Supporting Information

\title{
Establishing the Golden Range of Seebeck Coefficient for
}

\section{Maximizing Thermoelectric Performance}

Min Hong, ${ }^{\dagger}+\underset{+}{+}$ Wanyu Lyu,${ }^{\dagger}$ Yuan Wang, ${ }^{\dagger}$ Jin Zou, ${ }^{*},+, *$ and Zhi-Gang Chen ${ }^{*}, \dagger$,

$\dagger$ Centre for Future Materials, University of Southern Queensland, Springfield, Queensland 4300, Australia.

$\$$ School of Mechanical and Mining Engineering, The University of Queensland, Brisbane, Queensland 4072, Australia.

\# Centre for Microscopy and Microanalysis, The University of Queensland, Brisbane, Queensland 4072, Australia.

*Email: j.zou@uq.edu.au; zhigang.chen@usq.edu.au, zhigang.chen@uq.edu.au 


\section{Abbreviation and symbols}

$S \quad$ Seebeck coefficient

$\sigma \quad$ electrical conductivity

$\kappa \quad$ thermal conductivity

$\kappa_{\mathrm{e}} \quad$ electrical thermal conductivity

$\kappa_{1} \quad$ phonon thermal conductivity

$T \quad$ temperature

$S^{2} \sigma \quad$ power factor

$z T \quad$ figure of merit

$\beta \quad$ thermoelectric quality factor

$N_{\mathrm{v}} \quad$ band degeneracy

$m_{I}^{*} \quad$ inertial effective mass

$m_{\mathrm{d}}^{*} \quad$ density-of-states effective mass

M average atomic mass

$V \quad$ average atomic volume

$N \quad$ atomic number per primitive unit cell

$v_{\mathrm{m}} \quad$ mean speed of sound

$\gamma \quad$ Grüneisen parameter

$k_{\mathrm{B}} \quad$ Boltzmann constant

$E_{\mathrm{f}} \quad$ Fermi level

$E_{\mathrm{g}} \quad$ band gap

$\eta \quad$ reduced Fermi level, $\eta=E_{\mathrm{f}} / k_{\mathrm{B}} T$

$\eta^{\text {opt }} \quad$ optimal reduced Fermi level

$\eta_{\mathrm{PF}}^{\mathrm{opt}} \quad$ optimal reduced Fermi level for peak value of power factor

$\eta_{z \mathrm{~T}}^{\mathrm{opt}} \quad$ optimal reduced Fermi level for peak value of figure of merit

$\varepsilon \quad$ reduced band gap, $\varepsilon=E_{\mathrm{g}} / k_{\mathrm{B}} T$ 
$\sigma_{0} \quad$ weighted electrical conductivity

L Lorenz number

$E_{\text {def }} \quad$ deformation potential

CB conduction band

VB valence band

$S^{2} \sigma_{\max }$ maximum value of power factor

$z T_{\max } \quad$ maximum value of figure of merit

$R_{\mathrm{H}} \quad$ Hall coefficient

$n_{\mathrm{H}} \quad$ Hall carrier concentration

$\mu_{\mathrm{H}} \quad$ Hall carrier mobility

$f_{\mathrm{S}} \quad$ function describing the relation of Seebeck coefficient with Fermi level

$f_{\sigma} \quad$ function describing the relation of electrical conductivity with Fermi level

$f_{\mathrm{L}} \quad$ function describing the relation of Lorenz number with Fermi level 


\section{Big data survey result}

Table S1 Survey of reported maximum power factor $\left(S^{2} \sigma_{\max }\right)$, maximum $z T\left(z T_{\max }\right)$, the respectively corresponding Seebeck coefficient $(S)$ and temperature $(T)$.

\begin{tabular}{|c|c|c|c|c|c|c|c|}
\hline Materials & Method & $\begin{array}{l}S^{2} \sigma_{\max } \\
\left(10^{-4} \mathrm{Wm}^{-}\right. \\
\left.{ }^{1} \mathrm{~K}^{-2}\right)\end{array}$ & $\begin{array}{l}S \\
\left(\mu \mathrm{VK}^{-}\right. \\
\left.{ }^{1}\right)\end{array}$ & $T(\mathrm{~K})$ & $z T_{\max }$ & $\begin{array}{l}S \\
\left(\mu \mathrm{VK}^{-1}\right)\end{array}$ & $T(\mathrm{~K})$ \\
\hline \multicolumn{8}{|l|}{$\mathrm{GeSi}$} \\
\hline $\mathrm{Ge}_{0.3} \mathrm{Si}_{0.7^{1}}$ & ZL & 37.5 & -250 & 1200 & 1.1 & -265 & 1120 \\
\hline $\mathrm{GeSe}^{2}$ & BMA & 22.2 & 245 & 1073 & 1 & 245 & 1073 \\
\hline $\mathrm{GeSe}^{3}$ & BMA & 31.7 & -253 & 1123 & 1.3 & -253 & 1123 \\
\hline \multicolumn{8}{|l|}{ CoSb3 skutterudite } \\
\hline $\begin{array}{l}\mathrm{Yb}_{\mathrm{x}} \mathrm{Co}_{4} \mathrm{Sb}_{12} \quad \mathrm{x}=0.25 \\
0.3^{4}\end{array}$ & $\begin{array}{l}\mathrm{SSR}+\mathrm{H} \\
\mathrm{P}\end{array}$ & 43.6 & -163 & 700 & 1.3 & -173 & 850 \\
\hline $\operatorname{In}_{0.276} \mathrm{Co}_{4} \mathrm{Sb}_{11.9^{5}}$ & $\begin{array}{l}\mathrm{SSR}+\mathrm{H} \\
\mathrm{P}\end{array}$ & 43.5 & -222 & 600 & 1.2 & -233 & 750 \\
\hline $\mathrm{Ce}_{0.17} \mathrm{Co}_{4} \mathrm{Sb}_{12}{ }^{6}$ & $\begin{array}{l}\mathrm{SSR}+\mathrm{H} \\
\mathrm{P}\end{array}$ & 51 & -198 & 850 & 1.3 & $\begin{array}{ll}-198 \\
\end{array}$ & 850 \\
\hline $\mathrm{Yb}_{14} \mathrm{Mn}_{1.05} \mathrm{Sb}_{11}{ }^{7}$ & $\begin{array}{l}\text { SSR+S } \\
\text { PS }\end{array}$ & 8.66 & 208 & 1000 & 1.2 & 208 & 1000 \\
\hline $\begin{array}{l}\mathrm{Ba}_{0.3} \mathrm{In}_{0.3} \mathrm{Co}_{4} \mathrm{Sb}_{12-} \\
\left(\mathrm{BaFe}_{12} \mathrm{O}_{19}\right)_{0.0035}{ }^{8}\end{array}$ & $+\mathrm{SPS}$ & 45 & -200 & 800 & 1.64 & -218 & 850 \\
\hline $\begin{array}{l}\mathrm{Ba}_{0.3} \mathrm{In}_{0.3} \mathrm{Co}_{4} \mathrm{Sb}_{12-} \\
\mathrm{Co}_{0.002}(\mathrm{n}-)^{9}\end{array}$ & $+\mathrm{SPS}$ & 52.3 & -203 & 725 & 1.8 & -208 & 850 \\
\hline $\mathrm{Yb}_{0.3} \mathrm{Co}_{4} \mathrm{Sb}_{12.2}{ }^{10}$ & $\begin{array}{l}\mathrm{SSR}+\mathrm{H} \\
\mathrm{P}\end{array}$ & 54 & -209 & 673 & 1.46 & -212 & 825 \\
\hline $\begin{array}{l}\mathrm{In}_{0.2} \mathrm{Ce}_{0.15} \mathrm{Co}_{4} \mathrm{Sb}_{12} / \mathrm{In} \\
\mathrm{Sb}^{11}\end{array}$ & $\begin{array}{l}\mathrm{SSR}+\mathrm{M} \\
\mathrm{S}+\mathrm{SPS}\end{array}$ & 47 & -208 & 675 & 1.5 & -209 & 800 \\
\hline${ }_{2}^{\mathrm{Co} 4} \mathrm{Sb}_{11.3} \mathrm{Te}_{0.58} \mathrm{Se}_{0.12}{ }^{1}$ & $\begin{array}{l}\mathrm{SSR}+\mathrm{S} \\
\mathrm{PS}\end{array}$ & 40 & -209 & 775 & 1.11 & -210 & 800 \\
\hline $\begin{array}{l}\mathrm{Ba}_{0.08} \mathrm{La}_{0.05} \mathrm{Yb}_{0.04} \mathrm{Co}_{4} \\
\mathrm{Sb}_{12}{ }^{13}\end{array}$ & $\begin{array}{l}\text { SSR+S } \\
\text { PS }\end{array}$ & 50 & -182 & 700 & 1.7 & -193 & 850 \\
\hline
\end{tabular}




\begin{tabular}{|c|c|c|c|c|c|c|c|}
\hline \multicolumn{8}{|l|}{ half-Heusler } \\
\hline $\mathrm{FeNb}_{0.8} \mathrm{Ti}_{0.2} \mathrm{Sb}^{14}$ & $\begin{array}{l}\mathrm{LM}+\mathrm{SP} \\
\mathrm{S}\end{array}$ & 46.8 & 1000 & 194 & 1.1 & 204 & 1100 \\
\hline $\begin{array}{l}\mathrm{Fe}\left(\mathrm{V}_{0.6} \mathrm{Nb}_{0.4}\right)_{0.8} \mathrm{Ti}_{0.2} \mathrm{~S} \\
\mathrm{~b}^{15}\end{array}$ & $\begin{array}{l}\mathrm{LM}+\mathrm{SP} \\
\mathrm{S}\end{array}$ & 33.4 & 202 & 550 & 0.8 & 213 & 900 \\
\hline $\mathrm{Yb}_{14} \mathrm{Mn}_{0.2} \mathrm{Al}_{0.6} \mathrm{Sb}_{11}{ }^{16}$ & $\begin{array}{l}\mathrm{SSR}+\mathrm{M} \\
\mathrm{F}+\mathrm{HP}\end{array}$ & 7.0 & 230 & 1041 & 1.3 & 232 & 1223 \\
\hline $\mathrm{Yb}_{14} \mathrm{Mn}_{0.2} \mathrm{Al}_{0.8} \mathrm{Sb}_{11}{ }^{16}$ & $\begin{array}{l}\mathrm{SSR}+\mathrm{M} \\
\mathrm{F}+\mathrm{HP}\end{array}$ & 6.0 & 246 & 944 & 1.3 & 242 & 1223 \\
\hline $\mathrm{AgSb}_{0.96} \mathrm{Te}_{2} \mathrm{Zn}_{0.04}{ }^{17}$ & SSR & 17 & 290 & 580 & 1.9 & 290 & 580 \\
\hline $\mathrm{Nb}_{0.8} \mathrm{Ti}_{0.2} \mathrm{FeSb}^{18}$ & $\begin{array}{l}\mathrm{SSR}+\mathrm{H} \\
\mathrm{P}\end{array}$ & 56 & 155 & 672 & 1.1 & 198 & 1000 \\
\hline $\begin{array}{l}\mathrm{Ti}_{0.95} \mathrm{Mn}_{0.05} \mathrm{NiSn}_{0.95} \mathrm{~S} \\
\mathrm{~b}_{0.05}{ }^{19}\end{array}$ & $\begin{array}{l}\text { SSR } \\
\text { ingot }\end{array}$ & 31 & -141 & 778 & 0.45 & -141 & 867 \\
\hline \multicolumn{8}{|l|}{$\mathrm{SnSe}$} \\
\hline $\mathrm{SnSeTe}_{0.1}{ }^{20}$ & MSS & 6.77 & 295 & 450 & 1.1 & 323 & 800 \\
\hline $\mathrm{SnSe}^{21}$ & $\begin{array}{l}\mathrm{SSR}+\mathrm{S} \\
\mathrm{PS}\end{array}$ & 3.8 & 368 & 817 & 0.52 & 368 & 817 \\
\hline $\mathrm{SnSe}^{22}$ & $\begin{array}{l}\mathrm{SSR}+\mathrm{S} \\
\mathrm{PS}\end{array}$ & 2 & 380 & 790 & 0.57 & 380 & 790 \\
\hline $\mathrm{SnSe}_{0.87} \mathrm{~S}_{0.1} \mathrm{I}_{0.03}{ }^{23}$ & $\begin{array}{l}\text { SSR+ac } \\
\mathrm{HP}\end{array}$ & 3.9 & -640 & 770 & 1 & -640 & 770 \\
\hline $\operatorname{SnSeAg}_{0.01}{ }^{24}$ & $\begin{array}{l}\text { SSR+I } \\
\text { HP }\end{array}$ & 5.2 & 345 & 750 & 0.71 & 345 & 750 \\
\hline $\mathrm{SnSeNa}_{0.01}{ }^{25}$ & $\begin{array}{l}\mathrm{SSR}+\mathrm{S} \\
\mathrm{PS}\end{array}$ & 5.7 & 270 & 800 & 0.86 & 270 & 800 \\
\hline $\begin{array}{l}\mathrm{Sn}_{0.99} \mathrm{Na}_{0.01} \mathrm{Se}_{0.84} \mathrm{Te}_{0} . \\
16^{26}\end{array}$ & $\begin{array}{l}\text { BMA+ } \\
\text { SPS }\end{array}$ & 5 & 275 & 773 & 0.72 & 275 & 773 \\
\hline $\mathrm{Na}^{-S n S e}{ }^{27}$ & BS & 40.2 & 161 & 300 & 2 & 312 & 773 \\
\hline $\mathrm{SnSe}^{28}$ & BS & 9.86 & 342.9 & 823 & 2.6 & 339.9 & 923 \\
\hline $\mathrm{Sn}_{0.97} \mathrm{Na}_{0.03} \mathrm{Se}^{29}$ & BS & 28.1 & 162 & 317 & 2 & 250 & 800 \\
\hline \multicolumn{8}{|l|}{ SnTe } \\
\hline
\end{tabular}




\begin{tabular}{|c|c|c|c|c|c|c|c|}
\hline $\begin{array}{l}\mathrm{Sn}_{0.97} \mathrm{Bi}_{0.03} \mathrm{Te}-3 \% \\
\mathrm{SrTe}^{30}\end{array}$ & $\begin{array}{l}\text { SSR+S } \\
\text { PS }\end{array}$ & 26.5 & 121 & 472 & 1.2 & 170 & 800 \\
\hline $\begin{array}{l}\left(\mathrm{Sn}_{0.98} \mathrm{Bi}_{0.02} \mathrm{Te}\right)(\mathrm{HgT} \\
\mathrm{e})_{0.03}{ }_{31}\end{array}$ & $\begin{array}{l}\text { SSR+S } \\
\text { PS }\end{array}$ & 27 & 182 & 910 & 1.35 & 182 & 910 \\
\hline $\begin{array}{l}\left(\mathrm{Sn}_{0.91} \mathrm{Mn}_{0.14} \mathrm{Te}\right)\left(\mathrm{Cu}_{2}\right. \\
\mathrm{Te})_{0.05}{ }^{32}\end{array}$ & $\begin{array}{l}\text { SSR+S } \\
\text { PS }\end{array}$ & 18.2 & 221 & 802 & 1.6 & 207 & 923 \\
\hline $\begin{array}{l}\mathrm{SnCd}_{0.03} \mathrm{Te}- \\
(\mathrm{CdS})_{0.02} 33\end{array}$ & $\begin{array}{l}\text { SSR+S } \\
\text { PS }\end{array}$ & 18 & 200 & 900 & 1.3 & 205 & 873 \\
\hline $\begin{array}{l}\left(\mathrm{SnTe}_{0.994} \mathrm{I}_{0.006}\right)_{2.88}(\mathrm{In} \\
\left.{ }_{2} \mathrm{Te}_{2.982} \mathrm{I}_{0.018}\right)_{0.04}{ }^{34}\end{array}$ & $\begin{array}{l}\text { SSR+S } \\
\text { PS }\end{array}$ & 25 & 155 & 762 & 1.1 & 161 & 923 \\
\hline $\begin{array}{l}\mathrm{SnIn}_{0.015} \mathrm{Cd}_{0.015} \mathrm{Te}- \\
(\mathrm{CdS})_{0.03} 35\end{array}$ & $\begin{array}{l}\text { SSR+S } \\
\text { PS }\end{array}$ & 22 & 200 & 923 & 1.4 & 200 & 923 \\
\hline $\mathrm{SnMn}_{0.12} \mathrm{Te}^{36}$ & $\begin{array}{l}\text { SSR+S } \\
\text { PS }\end{array}$ & 23 & 212 & 762 & 1.3 & 226 & 900 \\
\hline $\begin{array}{l}\operatorname{AgSn}_{5} \mathrm{Sb}\left(\mathrm{Te}_{0.994} \mathrm{I}_{0.006}\right. \\
{ }_{37}\end{array}$ & $\begin{array}{l}\text { SSR+S } \\
\text { PS }\end{array}$ & 23.8 & 158 & 750 & 1.2 & 161 & 800 \\
\hline $\mathrm{SnTe}_{0.985 \mathrm{I}_{0.015}{ }^{38}}$ & $\begin{array}{l}\text { SSR+S } \\
\text { PS }\end{array}$ & 22 & 148 & 610 & 0.6 & 158 & 700 \\
\hline $\begin{array}{l}\mathrm{Sn}_{0.91} \mathrm{Mg}_{0.12} \mathrm{Te}\left(\mathrm{Cu}_{2} \mathrm{~T}\right. \\
\mathrm{e})_{0.05}^{39}\end{array}$ & $\begin{array}{l}\mathrm{SSR}+\mathrm{H} \\
\mathrm{P}\end{array}$ & 16 & 196 & 850 & 1.4 & 198 & 900 \\
\hline $\operatorname{In}_{0.0025} \mathrm{Sn}_{0.9975} \mathrm{Te}^{40}$ & $\begin{array}{l}\text { BMA+ } \\
\text { SPS }\end{array}$ & 21 & 162 & 873 & 1.1 & 162 & 873 \\
\hline $\mathrm{Sn}_{0.94} \mathrm{Cu}_{0.12} \mathrm{Te}^{41}$ & $\begin{array}{l}\mathrm{SSR}+\mathrm{H} \\
\mathrm{P}\end{array}$ & 18.5 & 155 & 850 & 1 & 155 & 850 \\
\hline $\mathrm{Sn}_{0.85} \mathrm{Mn}_{0.15} \mathrm{TeI}_{0.02}{ }^{42}$ & $\begin{array}{l}\mathrm{SSR}+\mathrm{H} \\
\mathrm{P}\end{array}$ & 17 & 242 & 900 & 1.3 & 242 & 900 \\
\hline $\mathrm{Sn}_{0.94} \mathrm{Mg}_{0.09} \mathrm{Te}^{43}$ & SSR & 30 & 165 & 860 & 1.2 & 165 & 860 \\
\hline \multicolumn{8}{|l|}{$\mathrm{PbTe}$} \\
\hline $\mathrm{PbTe}_{0.85} \mathrm{Se}_{0.15}{ }^{44}$ & $\begin{array}{l}\mathrm{SSR}+\mathrm{H} \\
\mathrm{P}\end{array}$ & 27.7 & 212 & 725 & 1.8 & 222 & 850 \\
\hline $\begin{array}{l}(\mathrm{PbTe})_{0.65}(\mathrm{PbS})_{0.25}(\mathrm{P} \\
\mathrm{bSe})_{0.1}\end{array}$ & $\begin{array}{l}\mathrm{SSR}+\mathrm{H} \\
\mathrm{P}\end{array}$ & 23.4 & 252 & 735 & 2 & 243 & 850 \\
\hline $\begin{array}{l}\mathrm{Na}_{0.007} \mathrm{PbSe} \\
\text { type })^{46}\end{array}$ & $\begin{array}{l}\mathrm{SSR}+\mathrm{H} \\
\mathrm{P}\end{array}$ & 15.3 & 176 & 761 & 1.3 & 200 & 850 \\
\hline
\end{tabular}




\begin{tabular}{|c|c|c|c|c|c|c|c|}
\hline $\begin{array}{l}\mathrm{Pb}_{0.92} \mathrm{Sr}_{0.08} \mathrm{Se} \\
\text { type })^{47}\end{array}$ & $\begin{array}{l}\mathrm{SSR}+\mathrm{H} \\
\mathrm{P}\end{array}$ & 12 & 210 & 696 & 1.5 & 260 & 900 \\
\hline $\mathrm{Pb}_{48}{ }_{1.002} \mathrm{Se}_{0.9982} \mathrm{Br}_{0.0018}$ & $\begin{array}{l}\mathrm{SSR}+\mathrm{H} \\
\mathrm{P}\end{array}$ & 24 & -133 & 530 & 1.2 & -207 & 850 \\
\hline $\mathrm{PbS}_{0.9978} \mathrm{Cl}_{0.0022}{ }^{49}$ & $\begin{array}{l}\mathrm{SSR}+\mathrm{H} \\
\mathrm{P}\end{array}$ & 17.5 & -138 & 515 & 0.7 & -205 & 850 \\
\hline $\mathrm{Pb}_{0.9975} \mathrm{Sb}_{0.0025} \mathrm{Se}^{50}$ & $\begin{array}{l}\mathrm{SSR}+\mathrm{H} \\
\mathrm{P}\end{array}$ & 24.5 & 170 & 500 & 1.45 & -87 & 830 \\
\hline${ }_{51}^{\mathrm{PbTe}}(\mathrm{SrTe})_{0.04} \mathrm{Na}_{0.02}$ & $\begin{array}{l}\text { SSR+S } \\
\text { PS }\end{array}$ & 27.8 & 243 & 742 & 2.2 & 284 & 915 \\
\hline $\begin{array}{l}2.5 \% \quad \text { K-doped } \\
\text { PbTe0.7S0.3 } \\
\text { type })^{52}\end{array}$ & $\begin{array}{l}\mathrm{SSR}+\mathrm{S} \\
\mathrm{PS}\end{array}$ & 21 & 219 & 520 & 2.3 & 304 & 850 \\
\hline $\begin{array}{l}\mathrm{Na}_{0.025} \mathrm{Eu}_{0.03} \mathrm{~Pb}_{0.945} \mathrm{~T} \\
\text { e(p-type })^{53}\end{array}$ & $\begin{array}{l}\text { SSR+S } \\
\text { PS }\end{array}$ & 26.3 & 197 & 488 & 2.1 & 226 & 850 \\
\hline $\begin{array}{l}\mathrm{Pb}_{0.95} \mathrm{SeSb}_{0.033} \\
\text { type })_{54}\end{array}$ & $\begin{array}{l}\text { SSR+S } \\
\text { PS }\end{array}$ & 8.2 & -190 & 638 & 1.67 & -201 & 900 \\
\hline $\begin{array}{l}\mathrm{PbIn}_{05} \ln _{05} \mathrm{Se} \quad(n \text {-type }) \\
\end{array}$ & & 17.5 & -200 & 680 & 1.2 & -250 & 880 \\
\hline $\begin{array}{l}\mathrm{Pb}_{0.9925} \mathrm{Cr}_{0.0075} \mathrm{Se}(n- \\
\text { type })_{56}\end{array}$ & $\begin{array}{l}\text { SSR+d } \\
\text { c HP }\end{array}$ & 28 & -160 & 300 & 1 & -263 & 573 \\
\hline $\begin{array}{l}\mathrm{Pb}_{0.995} \mathrm{Cr}_{0.005} \mathrm{Se}(n- \\
\text { type })^{56}\end{array}$ & $\begin{array}{l}\text { SSR+d } \\
\text { c HP }\end{array}$ & 31 & -158 & 300 & 1 & -275 & 673 \\
\hline $\begin{array}{l}\mathrm{PbTe}_{0.9988} \mathrm{I}_{0.0012}(n- \\
\text { type }) 57\end{array}$ & $\begin{array}{l}\text { SSR+ } \\
\mathrm{HP}\end{array}$ & 29 & -162 & 531 & 1.4 & -215 & 730 \\
\hline $\begin{array}{l}\mathrm{PbS}\left(\mathrm{Bi}_{2} \mathrm{~S}_{3}\right)_{0.01}\left(\mathrm{PbCl}_{2}\right. \\
)_{0.01}(n \text {-type })\end{array}$ & $\begin{array}{l}\mathrm{SSR}+\mathrm{S} \\
\mathrm{PS}\end{array}$ & 13.5 & -225 & 923 & 1.1 & -225 & 923 \\
\hline $\begin{array}{l}\left(\mathrm{Pb}_{0.98} \mathrm{Na}_{0.02} \mathrm{Te}\right)(\mathrm{Mg} \\
\mathrm{Te})_{0.06} 59\end{array}$ & $\begin{array}{l}\mathrm{SSR}+\mathrm{S} \\
\mathrm{PS}\end{array}$ & 26 & 246 & 475 & 2 & 298 & 823 \\
\hline $\begin{array}{l}\left(\mathrm{Pb}_{0.98} \mathrm{Na}_{0.02} \mathrm{Se}\right)- \\
(\mathrm{CdS})_{0.03} 60\end{array}$ & $\begin{array}{l}\text { SSR+S } \\
\text { PS }\end{array}$ & 17 & 240 & 900 & 1.6 & 250 & 923 \\
\hline \multicolumn{8}{|l|}{ GeTe } \\
\hline $\begin{array}{l}(\mathrm{GeTe})_{0.937} \\
\left(\mathrm{Bi}_{2} \mathrm{Se}_{0.2} \mathrm{Te}_{0.8}\right)_{0.063}{ }^{61}\end{array}$ & $\begin{array}{l}\mathrm{SSR}+\mathrm{S} \\
\mathrm{PS}\end{array}$ & 63.5 & 216 & 723 & 2.2 & 216 & 723 \\
\hline
\end{tabular}




\begin{tabular}{|c|c|c|c|c|c|c|c|}
\hline $\mathrm{Ge}_{0.9} \mathrm{Sb}_{0.1} \mathrm{Te}^{62}$ & $\begin{array}{l}\text { SSR, } \\
\text { ingot }\end{array}$ & 51.4 & 256 & 725 & 1.9 & 256 & 725 \\
\hline $\mathrm{Ge}_{0.935} \mathrm{Bi}_{0.065} \mathrm{Te}^{63}$ & $\begin{array}{l}\text { SSR+S } \\
\text { PS }\end{array}$ & 34 & 242 & 677 & 1.8 & 236 & 700 \\
\hline $\mathrm{Ge}_{64} \mathrm{Sb}_{0.1} \mathrm{Te}_{0.88} \mathrm{Se}_{0.12}$ & $\begin{array}{l}\text { SSR+S } \\
\text { PS }\end{array}$ & 31.4 & 230 & 618 & 2 & 238 & 700 \\
\hline $\begin{array}{l}\mathrm{Ge}_{0.9} \mathrm{Sb}_{0.1} \mathrm{Te}_{0.9} \\
\mathrm{Se}_{0.05} \mathrm{~S}_{0.05} 65\end{array}$ & $\begin{array}{l}\text { SSR+S } \\
\text { PS }\end{array}$ & 43 & 260 & 678 & 2 & 260 & 678 \\
\hline $\mathrm{Ge}_{0.9} \mathrm{Cd}_{0.05} \mathrm{Bi}_{0.05} \mathrm{Te}^{66}$ & $\begin{array}{l}\text { SSR+S } \\
\text { PS }\end{array}$ & 41 & 231 & 650 & 2.3 & 231 & 650 \\
\hline $\mathrm{Ge}_{7} \mathrm{Sb}_{2} \mathrm{Te}_{20}+\mathrm{Sb}_{0.195}{ }^{6}$ & $\begin{array}{l}\text { SSR+S } \\
\text { PS }\end{array}$ & 36.3 & 241 & 615 & 2.2 & 238 & 763 \\
\hline $\mathrm{Ge}_{0.89} \mathrm{Sb}_{0.1} \operatorname{In}_{0.01} \mathrm{Te}^{68}$ & $\begin{array}{l}\mathrm{SSR}+\mathrm{S} \\
\mathrm{PS}\end{array}$ & 38 & 260 & 650 & 2.3 & 256 & 736 \\
\hline $\begin{array}{l}\mathrm{Ge}_{0.87} \mathrm{~Pb}_{0.13} \mathrm{Te} \\
\left(\mathrm{Bi}_{2} \mathrm{Te}_{3}\right)_{0.03} 69\end{array}$ & $\begin{array}{l}\mathrm{SSR}+\mathrm{S} \\
\mathrm{PS}\end{array}$ & 35 & 270 & 770 & 1.9 & 270 & 770 \\
\hline$\left.{ }_{0}^{(\mathrm{GeTe}}\right)_{0.73}(\mathrm{PbSe})_{0.27^{7}}$ & $\begin{array}{l}\mathrm{SSR}+\mathrm{S} \\
\mathrm{PS}\end{array}$ & 21.4 & 245 & 800 & 2.3 & 245 & 800 \\
\hline $\mathrm{Ge}_{0.87} \mathrm{~Pb}_{0.13} \mathrm{Te}^{71}$ & $\begin{array}{l}\mathrm{SSR}+\mathrm{S} \\
\mathrm{PS}\end{array}$ & 44.3 & 192 & 612 & 2.3 & 195 & 687 \\
\hline $\mathrm{Ge}_{0.76} \mathrm{Sb}_{0.08} \mathrm{~Pb}_{0.12} \mathrm{Te}^{72}$ & $\begin{array}{l}\mathrm{SSR}+\mathrm{S} \\
\mathrm{PS}\end{array}$ & 31.6 & 232 & 575 & 2.3 & 265 & 800 \\
\hline${ }_{5} \mathrm{Ge}_{0.87} \mathrm{~Pb}_{0.13} \mathrm{Bi}_{0.1} \mathrm{Te}_{1.1}$ & $\begin{array}{l}\mathrm{SSR}+\mathrm{S} \\
\mathrm{PS}\end{array}$ & 24.7 & 226 & 603 & 2.1 & 242 & 690 \\
\hline $\mathrm{Ge}_{0.86} \mathrm{~Pb}_{0.1} \mathrm{Bi}_{0.04} \mathrm{Te}^{74}$ & $\begin{array}{l}\text { SSR+S } \\
\text { PS }\end{array}$ & 30 & 271 & 569 & 2.4 & 287 & 600 \\
\hline $\mathrm{Ge}_{0.86} \mathrm{Mn}_{0.1} \mathrm{Sb}_{0.04} \mathrm{Te}^{75}$ & $\begin{array}{l}\text { SSR+S } \\
\text { PS }\end{array}$ & 30.2 & 176 & 667 & 1.61 & 185 & 823 \\
\hline $\mathrm{Ge}_{0.98} \operatorname{In}_{0.02} \mathrm{Te}^{76}$ & $\begin{array}{l}\text { SSR+S } \\
\text { PS }\end{array}$ & 42.3 & 190 & 660 & 1.3 & 191 & 628 \\
\hline $\begin{array}{l}(\mathrm{GeTe})_{0.8} \\
\left(\mathrm{AgSbTe}_{2}\right)_{0.2} 77\end{array}$ & $\begin{array}{l}\text { SSR+S } \\
\text { PS }\end{array}$ & 33 & 211 & 773 & 1.75 & 211 & 773 \\
\hline $\begin{array}{l}\left(\mathrm{Ge}_{0.98} \mathrm{Dy}_{0.02} \mathrm{Te}\right)_{0.85} \\
\left(\mathrm{AgSbTe}_{2}\right)_{0.15}{ }^{78}\end{array}$ & $\begin{array}{l}\text { SSR } \\
\text { ingot }\end{array}$ & 28 & 195 & 750 & 1.5 & 195 & 750 \\
\hline
\end{tabular}




\begin{tabular}{|c|c|c|c|c|c|c|c|}
\hline $\begin{array}{l}\left(\mathrm{Ge}_{0.99} \mathrm{Yb}_{0.01} \mathrm{Te}\right)_{0.85} \\
\left(\mathrm{AgSbTe}_{2}\right)_{0.15}{ }^{79}\end{array}$ & $\begin{array}{l}\text { SSR } \\
\text { ingot }\end{array}$ & 35 & 196 & 700 & 1.5 & 205 & 773 \\
\hline $\begin{array}{l}\left(\mathrm{Ge}_{0.99} \mathrm{Ce}_{0.01} \mathrm{Te}\right)_{0.85} \\
\left(\mathrm{AgSbTe}_{2}\right)_{0.15}{ }^{79}\end{array}$ & $\begin{array}{l}\text { SSR } \\
\text { ingot }\end{array}$ & 36 & 198 & 700 & 1.5 & 208 & 773 \\
\hline \multicolumn{8}{|l|}{ Mg-alloys } \\
\hline $\mathrm{Mg}_{2} \mathrm{Sn}_{0.75} \mathrm{Ge}_{0.25}{ }^{80}$ & $\begin{array}{l}\mathrm{BMA}+ \\
\text { dc HP }\end{array}$ & 55.1 & -206 & 623 & 1.4 & -217 & 723 \\
\hline $\mathrm{Mg}_{2} \mathrm{Si}_{0.3} \mathrm{Sn}_{0.7} \mathrm{Sb}_{0.006}{ }^{81}$ & $\begin{array}{l}\mathrm{SSR}+\mathrm{H} \\
\mathrm{P}\end{array}$ & 47.8 & -213 & 550 & 1.3 & -235 & 700 \\
\hline 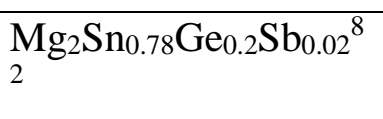 & $\begin{array}{l}\mathrm{BMA}+ \\
\mathrm{HP}\end{array}$ & 53 & -210 & 573 & 1.4 & -227 & 723 \\
\hline $\begin{array}{l}\text { Sb-doped } \\
\mathrm{Mg}_{2} \mathrm{Si}_{0.5} \mathrm{Sn}_{0.5}{ }^{83}\end{array}$ & $\begin{array}{l}\text { Melting } \\
+ \text { SPS }\end{array}$ & 26.3 & -227 & 563 & 1.63 & -235 & 615 \\
\hline $\begin{array}{l}\text { Sb-doped } \\
\mathrm{Mg}_{2} \mathrm{Si}_{0.3} \mathrm{Sn}_{0.7}{ }^{84}\end{array}$ & $\begin{array}{l}\mathrm{SSR}+\mathrm{P} \\
\mathrm{AS}\end{array}$ & 44 & -190 & 510 & 1.2 & -220 & 750 \\
\hline $\begin{array}{l}\text { Sb-doped } \\
\mathrm{Mg}_{2} \mathrm{Si}_{0.4} \mathrm{Sn}_{0.6}{ }^{85}\end{array}$ & $\begin{array}{l}\text { Melting } \\
\text { ingot }\end{array}$ & 43.6 & -198 & 630 & 1 & -215 & 760 \\
\hline $\begin{array}{l}\text { Sb-doped } \\
\mathrm{Mg}_{2} \mathrm{Si}_{0.6} \mathrm{Sn}_{0.4}{ }^{85}\end{array}$ & $\begin{array}{l}\text { Melting } \\
\text { ingot }\end{array}$ & 94.6 & -217 & 890 & 1.1 & -213 & 850 \\
\hline $\begin{array}{l}\mathrm{Mg}_{3} \mathrm{Sb}_{1.48} \\
\mathrm{Bi}_{0.48} \mathrm{Te}^{86}\end{array}$ & $\begin{array}{l}\text { Arc } \\
\text { melting } \\
+ \text { SPS }\end{array}$ & 14 & 238 & 500 & 1.65 & -260 & 725 \\
\hline $\begin{array}{l}\mathrm{Mg}_{87}{ }_{89} \mathrm{Li}_{0.01} \mathrm{Ag}_{0.97} \mathrm{Sb}_{0} \\
.99\end{array}$ & $\begin{array}{l}\text { BMA+ } \\
\mathrm{PH}\end{array}$ & 25 & 196 & 350 & 1.2 & 189 & 525 \\
\hline $\mathrm{Mg}_{3} \mathrm{Sb}_{0.6} \mathrm{Bi}_{1.4}{ }^{88}$ & $\begin{array}{l}\mathrm{SSR}+\mathrm{H} \\
\mathrm{P}\end{array}$ & 23 & -190 & 564 & 1.2 & -195 & 500 \\
\hline \multicolumn{8}{|l|}{$\mathrm{Bi}_{2} \mathrm{Te}_{3}$} \\
\hline$(\mathrm{Bi}, \mathrm{Sb})_{2} \mathrm{Te}_{3}{ }^{89}$ & $\begin{array}{l}\text { BMA+ } \\
\text { SPS }\end{array}$ & 43 & 227 & 300 & 1.38 & 231 & 323 \\
\hline $\mathrm{Bi}_{2} \mathrm{Te}_{3}{ }^{90}$ & $\begin{array}{l}\mathrm{MSS}+\mathrm{C} \\
\mathrm{P}\end{array}$ & 14.5 & -192 & 300 & 0.91 & -198 & 350 \\
\hline $\mathrm{Bi}_{0.5} \mathrm{Sb}_{1.5} \mathrm{Te}_{3}{ }^{90}$ & $\begin{array}{l}\mathrm{MSS}+\mathrm{C} \\
\mathrm{P}\end{array}$ & 17.3 & 224 & 300 & 1.2 & 234 & 363 \\
\hline $\mathrm{Bi}_{0.5} \mathrm{Sb}_{1.5} \mathrm{Te}_{3}{ }^{91}$ & $\begin{array}{l}\text { MSS+S } \\
\text { PS }\end{array}$ & 28.3 & 196 & 300 & 1.2 & 200 & 320 \\
\hline
\end{tabular}




\begin{tabular}{|c|c|c|c|c|c|c|c|}
\hline 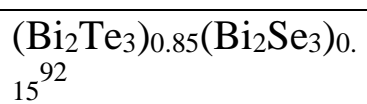 & $\begin{array}{l}\text { SG+SP } \\
\text { S }\end{array}$ & 12 & -216 & 400 & 0.71 & -213 & 480 \\
\hline $\mathrm{Bi}_{0.5} \mathrm{Sb}_{1.5} \mathrm{Te}_{3}{ }^{93}$ & $\begin{array}{l}\mathrm{MSS}+\mathrm{S} \\
\text { PS }\end{array}$ & 32 & 222 & 300 & 1.13 & 235 & 360 \\
\hline $\mathrm{Bi}_{0.5} \mathrm{Sb}_{1.5} \mathrm{Te}_{3}{ }^{94}$ & $\begin{array}{l}\mathrm{BM}+\mathrm{H} \\
\mathrm{P}\end{array}$ & 44 & 188 & 300 & 1.4 & 210 & 373 \\
\hline $\mathrm{Bi}_{0.5} \mathrm{Sb}_{1.5} \mathrm{Te}_{3}{ }^{95}$ & $\begin{array}{l}\text { BMA+ } \\
\mathrm{HP}\end{array}$ & 43 & 188 & 298 & 1.3 & 210 & 373 \\
\hline $\mathrm{Bi}_{2} \mathrm{Te}_{2.7} \mathrm{Se}_{0.3}{ }^{96}$ & BS & 58.6 & 167 & 300 & 1.18 & 190 & 410 \\
\hline $\begin{array}{l}\mathrm{Bi}_{2}\left(\mathrm{Te}_{1-\mathrm{x}} \mathrm{Se}_{\mathrm{x}}\right)_{3-} \\
\mathrm{I}(0.08 \%)^{97}\end{array}$ & $\mathrm{ZM}$ & 54.8 & -195 & 300 & 1.1 & -202 & 340 \\
\hline $\mathrm{Bi}_{0.52} \mathrm{Sb}_{1.48} \mathrm{Te}_{3}{ }^{98}$ & $\begin{array}{l}\mathrm{MS}+\mathrm{SP} \\
\mathrm{S}\end{array}$ & 35.1 & 227 & 300 & 1.56 & 227 & 300 \\
\hline $\mathrm{Cu}_{0.01} \mathrm{Bi}_{2} \mathrm{Te}_{2.7} \mathrm{Se}_{0.3}{ }^{99}$ & $\begin{array}{l}\text { BMA+ } \\
\text { dc HP }\end{array}$ & 31.5 & -188.5 & 300 & 1.1 & -193.7 & 373 \\
\hline $\mathrm{Bi}_{2} \mathrm{Te}_{2.79} \mathrm{Se}_{0.21}{ }^{100}$ & $\begin{array}{l}\mathrm{HP}+\mathrm{H} \\
\mathrm{D}\end{array}$ & 41.6 & -187 & 300 & 1.2 & -200 & 357 \\
\hline $\mathrm{Bi}_{2} \mathrm{Te}_{2.7} \mathrm{Se}_{0.3}{ }^{101}$ & $\begin{array}{l}\text { BMA+ } \\
\text { dc HP }\end{array}$ & 35.1 & -190 & 396 & 1.04 & -207 & 398 \\
\hline
\end{tabular}

The abbreviations used in the column of preparation method represent the following meanings: $\mathrm{ZL}=$ zone leveling, $\mathrm{MSS}=$ microwave solvothermal, $\mathrm{SSR}=$ solid state reaction, $\mathrm{LM}=$ levitation melting, $\mathrm{CP}=$ cold pressing, $\mathrm{SG}=$ solution grow, $\mathrm{SPS}=$ spark plasma sintering, $\mathrm{HP}=$ hot pressing, dc $\mathrm{HP}=$ direct current hot pressing, $\mathrm{IHP}=$ induction heating hot pressing, $\mathrm{PAS}=$ plasma activated sintering, $\mathrm{MF}=$ metal flux, $\mathrm{BMA}=$ ball milling alloy, $\mathrm{MA}=$ melting alloy, $\mathrm{HPS}=$ high pressure synthesis, $\mathrm{BS}=$ Bridgman-Stockbarger, $\mathrm{BM}=$ ball milling, $\mathrm{THM}=$ travelling heater method, MS = melt spinning, Te-MS = Te-rich melt spinning, $\mathrm{ZM}=$ zone melting.

\section{Details of modeling study based on the Kane energy relation}

Thermoelectric properties of single-band Kane model are given by ${ }^{102}$

Seebeck coefficient

$$
S=\frac{k_{B}}{e}\left[\frac{F_{1,-2}^{1}(\eta, \beta)}{F_{1,-2}^{0}(\eta, \beta)}-\eta\right] \text {, }
$$

Hall carrier concentration 


$$
n_{H}=\frac{N\left(2 m_{b}^{*} k_{B} T\right)^{\frac{3}{2}}}{3 \pi^{2} \hbar^{3}} \frac{F_{3 / 2,0}^{0}(\eta, \beta)}{A}
$$

Hall carrier mobility

$$
\mu_{H}=A \frac{2 \pi \hbar^{4} e C_{l}}{m_{I}^{*}\left(2 m_{b}^{*} k_{B} T\right)^{3 / 2} E_{d e f}^{2}} \frac{3 F_{1,-2}^{0}(\eta, \beta)}{F_{3 / 2,0}^{0}(\eta, \beta)}
$$

Hall factor

$$
A=\frac{3 K(K+2)}{(2 K+1)^{2}} \frac{F_{1 / 2,-4}^{0} F_{3 / 2,0}^{0}}{\left(F_{1,-2}^{0}\right)^{2}}
$$

Electrical conductivity

$$
\sigma=n_{H} \mu_{H} e=\frac{2 e^{2} N \hbar C_{l}}{\pi m_{I}^{*} E_{d e f}^{2}} F_{1,2}^{0}(\eta, \beta)
$$

Generalized Fermi integration

$$
F_{m, k}^{n}(\eta, \beta)=\int_{0}^{\infty}\left[-\frac{\partial f(\eta)}{\partial \varepsilon}\right] \varepsilon^{n}\left(\varepsilon+\beta \varepsilon^{2}\right)^{m}\left[(1+2 \beta \varepsilon)^{2}+2\right]^{\frac{k}{2}} d \varepsilon
$$

Electrical thermal conductivity is calculated according to the Wiedemann-Franz law, namely

$$
\kappa_{e}=L \sigma T
$$

with $L$ representing the Lorenz number, and give by

$$
L=\left(\frac{k_{B}}{e}\right)^{2}\left[\frac{F_{1,-2}^{2}(\eta, \beta)}{F_{1,-2}^{0}(\eta, \beta)}-\left(\frac{F_{1,-2}^{1}(\eta, \beta)}{F_{1,-2}^{0}(\eta, \beta)}\right)^{2}\right] .
$$

In the above equations, $\eta$ is the reduced Fermi level, $\beta=\frac{k_{B} T}{E_{g}}\left(E_{g}\right.$ is the band gap) is the reciprocal reduced band gap, $k_{B}$ is Boltzmann constant, $e$ is the elementary charge, $N$ is the 
band degeneracy, $\hbar$ is the reduced Planck constant, $C_{l}$ is the combination of elastic constants, $m_{I}^{*}$ is the inertial effective mass, and $E_{\text {def }}$ is the deformation potential, respectively ${ }^{103-104}$.

Considering both conduction band (CB) and valence band (VB), the total thermoelectric properties are calculated using the following equations.

Total electrical conductivity

$$
\sigma_{\text {tot }}=\sigma_{C}+\sigma_{V}
$$

Total Seebeck coefficient

$$
S_{t o t}=\frac{S_{C} \sigma_{C}+S_{V} \sigma_{V}}{\sigma_{C}+\sigma_{V}}
$$

Total Lorenz number

$$
L_{t o t}=\frac{L_{C} \sigma_{C}+L_{V} \sigma_{V}}{\sigma_{C}+\sigma_{V}}
$$

Total Hall coefficient

$$
R_{H_{\text {tot }}}=\frac{R_{H_{C}} \sigma_{C}^{2}+R_{H_{V}} \sigma_{V}^{2}}{\left(\sigma_{C}+\sigma_{V}\right)^{2}}
$$

Total Hall carrier concentration

$$
n_{H_{t o t}}=\frac{1}{e R_{H_{t o t}}} \text {, }
$$

Total Hall carrier mobility

$$
\mu_{H_{t o t}}=\sigma_{t o t} R_{H_{t o t}} .
$$

In the above equations, the tensors of thermoelectric properties are labeled with the subscript of $\mathrm{C}$ for $\mathrm{CB}$ and a subscript of $\mathrm{V}$ for $\mathrm{VB}$. 


\section{Modeling of phonon transport}

According to the Debye-Callaway model, ${ }^{105-106} \kappa_{l}$ can be calculated by

$$
\kappa_{l}=\frac{k_{B}}{2 \pi^{2} v}\left(\frac{k_{B} T}{\hbar}\right)^{3} \int_{0}^{\frac{\theta_{D}}{T}} \tau_{t o t} \frac{z^{4} \exp (z)}{[\exp (z)-1]^{2}} d z
$$

The integrand item in conjunction with the coefficient of Equation (S15) is the spectral lattice thermal conductivity $\left(\kappa_{s}\right)^{107}$, namely

$$
\kappa_{s}=\frac{k_{B}}{2 \pi^{2} v}\left(\frac{k_{B} T}{\hbar}\right)^{3} \tau_{t o t} \frac{z^{4} \exp (z)}{[\exp (z)-1]^{2}}
$$

In the above equation, $z=\frac{\hbar \omega}{k_{B} T}$ (with $\omega$ denoting the phonon frequency) is the reduced phonon frequency, $\hbar$ is the reduced Plank constant, $\theta_{D}$ is the Debye temperature, $v=\left[\frac{1}{3}\left(\frac{1}{v_{L}^{3}}+\frac{2}{v_{T}^{3}}\right)\right]^{-1 / 3}$ (with $U_{L}$ and $U_{T}$ respectively denoting the longitudinal and transverse sound velocities) is the sound velocity, and $\tau_{\text {tot }}$ is the total relaxation time. The phonon scattering pathways generally include phonon-phonon Umklapp (U), electron-phonon (E), point defects (PD), and grain boundaries (B). ${ }^{107-108}$ The relevant phonon relaxation times are given by

Umklapp phonon scattering

$$
\tau_{U}^{-1}=(\beta+1) \frac{\hbar \gamma^{2} \omega^{2} T}{\bar{M} v^{2} \theta_{D}} \exp \left(-\frac{\theta_{D}}{3 T}\right)
$$

Point defect phonon scattering

$$
\tau_{P D}^{-1}=\frac{\bar{V} \omega^{4}}{4 \pi v^{3}} \Gamma, \text { and }
$$

Grain boundary phonon scattering 


$$
\tau_{B}^{-1}=\frac{v}{d}
$$

For material with dislocations, the scattering caused by the dislocations (D) should be considered, which includes dislocation core (DC) and dislocation strain (DS). ${ }^{109}$ The relaxation time of dislocation scattering can be considered as

Dislocation core phonon scattering

$$
\tau_{D C}^{-1}=N_{D} \frac{\bar{V}^{4 / 3}}{v^{2}} \omega^{3}, \text { and }
$$

Dislocation strain phonon scattering

$$
\tau_{D S}^{-1}=0.6 B_{D}^{2} N_{D} \gamma^{2} \omega\left\{\frac{1}{2}+\frac{1}{24}\left(\frac{1-2 r}{1-r}\right)^{2}\left[1+\sqrt{2}\left(\frac{v_{\|}}{v_{\perp}}\right)\right]^{2}\right\}
$$

where $N_{D}$ is the dislocation density, $B_{D}$ is the effective Burger's vector, $r$ is the Poisson's ratio.

Nano precipitates phonon scattering 53,110

$$
\tau_{n p}^{-1}=v\left[(2 \pi R)^{-1}+\left(\pi R^{2} \frac{4}{9}\left(\frac{\Delta D}{D}\right)^{2}\left(\frac{\omega R}{v}\right)^{4}\right)^{-1}\right]^{-1} N_{p}
$$

In the above equations, $\gamma$ is the Grüneisen parameter, $\beta$ is the ratio between the normal process and Umklapp phonon scattering, $\rho$ is the sample density, $\bar{V}$ is the average atomic volume, $\bar{M}$ is the average atomic mass, $\Gamma$ is the point defect scattering parameter, $d$ is the grain size, $a$ is the lattice parameter, and $N_{s}$ is the number of stacking faults crossing a line of unit length, $R$ is the average radius for the precipitates $D$ is the matrix density, $\Delta D$ is the density difference between the precipitate and matrix, $N_{p}$ is the number density of precipitates, respectively. The phonon relaxation time of phonons scattered by nanoscale pores is calculated by Equation (S22) by assuming $\Delta D$ equal to $D$. 
Table S2. Physical properties used to calculate $\kappa_{l}$ based on various phonon scattering processes.

\begin{tabular}{ll}
\hline Parameters & Values \\
\hline Debye temperature $\theta_{D}(\mathrm{~K})$ & $156^{111}$ \\
Longitudinal sound velocity $v_{L}\left(\mathrm{~ms}^{-1}\right)$ & $3150^{54}$ \\
Transverse sound velocity $v_{T}\left(\mathrm{~ms}^{-1}\right)$ & $1600^{54}$ \\
Sound velocity $v\left(\mathrm{~ms}^{-1}\right)$ & $1787^{54}$ \\
Average atomic mass $\bar{M}(\mathrm{~kg})$ & $2.66 \times 10^{-25}$ \\
Sample density $D\left(\mathrm{~g} \mathrm{~cm}{ }^{-3}\right)$ & $7.91(\mathrm{Exp})$. \\
Grain size $d(\mu \mathrm{m})$ & $2.2(\mathrm{Exp})$. \\
Magnitude of Burger's vector $B_{D}(\AA)$ & $4.8(\mathrm{Exp})$. \\
Poisson's ratio $r$ & $0.243{ }^{112}$ \\
Grüneisen parameter $\gamma$ & $1.7^{112}$ \\
Bulk modulus $K(\mathrm{GPa})$ & 37.4 \\
Number density of nanoscale pores with size $<500 \mathrm{~nm}\left(\mathrm{~m}^{-3}\right)$ & $1.3 \times 10^{17}$ \\
\hline
\end{tabular}




\section{Phase Examination of $\mathrm{Pb}_{1-x} \mathrm{Bi}_{\mathrm{x}} \mathrm{Se}$}

Figure $\mathrm{S} 1$ is $\mathrm{XRD}$ patterns taken from as-synthesized $\mathrm{Pb}_{1-\mathrm{x}} \mathrm{Bi}_{\mathrm{x}} \mathrm{Se}$. Compared with the standard XRD pattern of PbSe (JCPDS 65-1074) ${ }^{113}$, no detectable impurity is found in the assynthesized products.

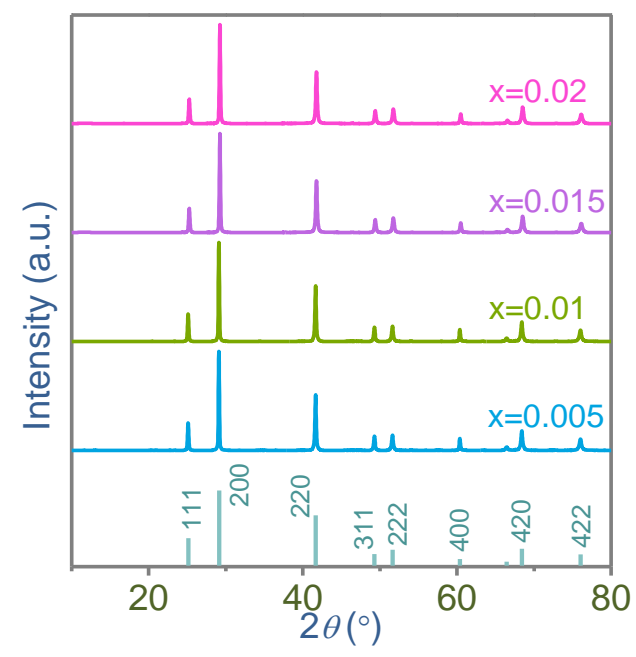

Figure $\mathrm{S} 1 \mathrm{XRD}$ patterns collected from $\mathrm{Pb}_{1-\mathrm{x}} \mathrm{Bi}_{\mathrm{x}} \mathrm{Se}$. 


\section{Determination of $\kappa_{e}$ of $\mathrm{Pb}_{1-\mathrm{x}} \mathrm{Bi}_{\mathrm{x}} \mathrm{Se}$}

Figure $\mathrm{S} 2$ shows the determined $\kappa_{e}$ of $\mathrm{Pb}_{1-\mathrm{x}} \mathrm{Bi}_{\mathrm{x}} \mathrm{Se}$ based on the Wiedemann-Franz law.

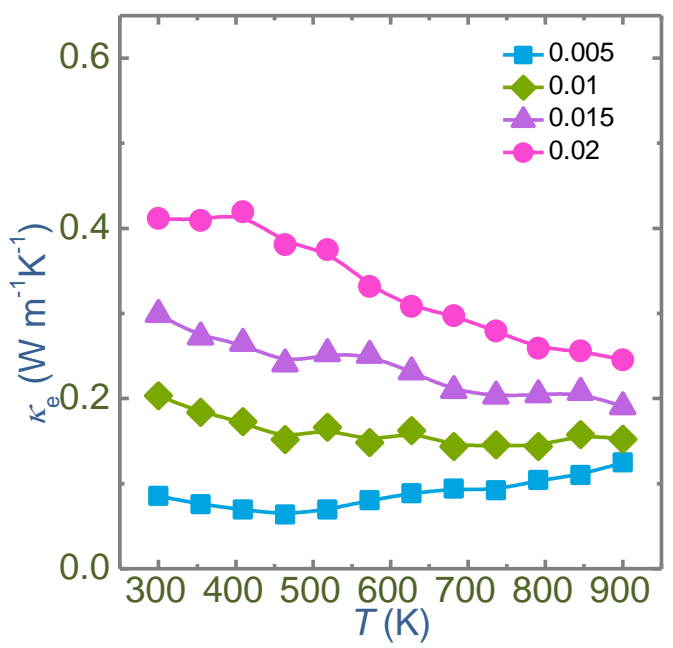

Figure $\mathrm{S} 2$ Calculated $\kappa_{e}$ of $\mathrm{Pb}_{1-\mathrm{x}} \mathrm{Bi}_{\mathrm{x}} \mathrm{Se}$ according to Wiedemann-Franz law. 
7. Comparison of achieved $z T$ with the reported values

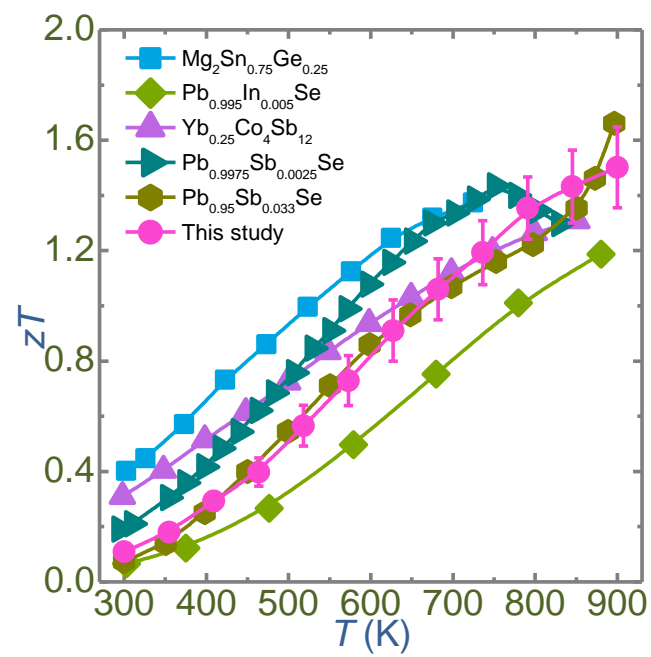

Figure S3 Comparison of (a) temperature-dependent peak $z T$ of $\mathrm{Pb}_{0.98} \mathrm{Bi}_{0.02} \mathrm{Se}$ in this study with the reported $n$-type cutting-edge thermoelectric materials of $\mathrm{Mg}_{2} \mathrm{Sb}_{0.75} \mathrm{Ge}_{0.25},{ }^{80}$ $\mathrm{Pb}_{0.995} \mathrm{In}_{0.005} \mathrm{Se},{ }^{55} \mathrm{Yb}_{0.25} \mathrm{Co}_{4} \mathrm{Sb}_{12},{ }^{4} \mathrm{~Pb}_{0.9975} \mathrm{Sb}_{0.0025} \mathrm{Se},{ }^{50}$ and $\mathrm{Pb}_{0.95} \mathrm{Sb}_{0.033} \mathrm{Se}^{54}$ 


\section{Relationships between peaks of $S^{2} \sigma, z T$ and $S$}
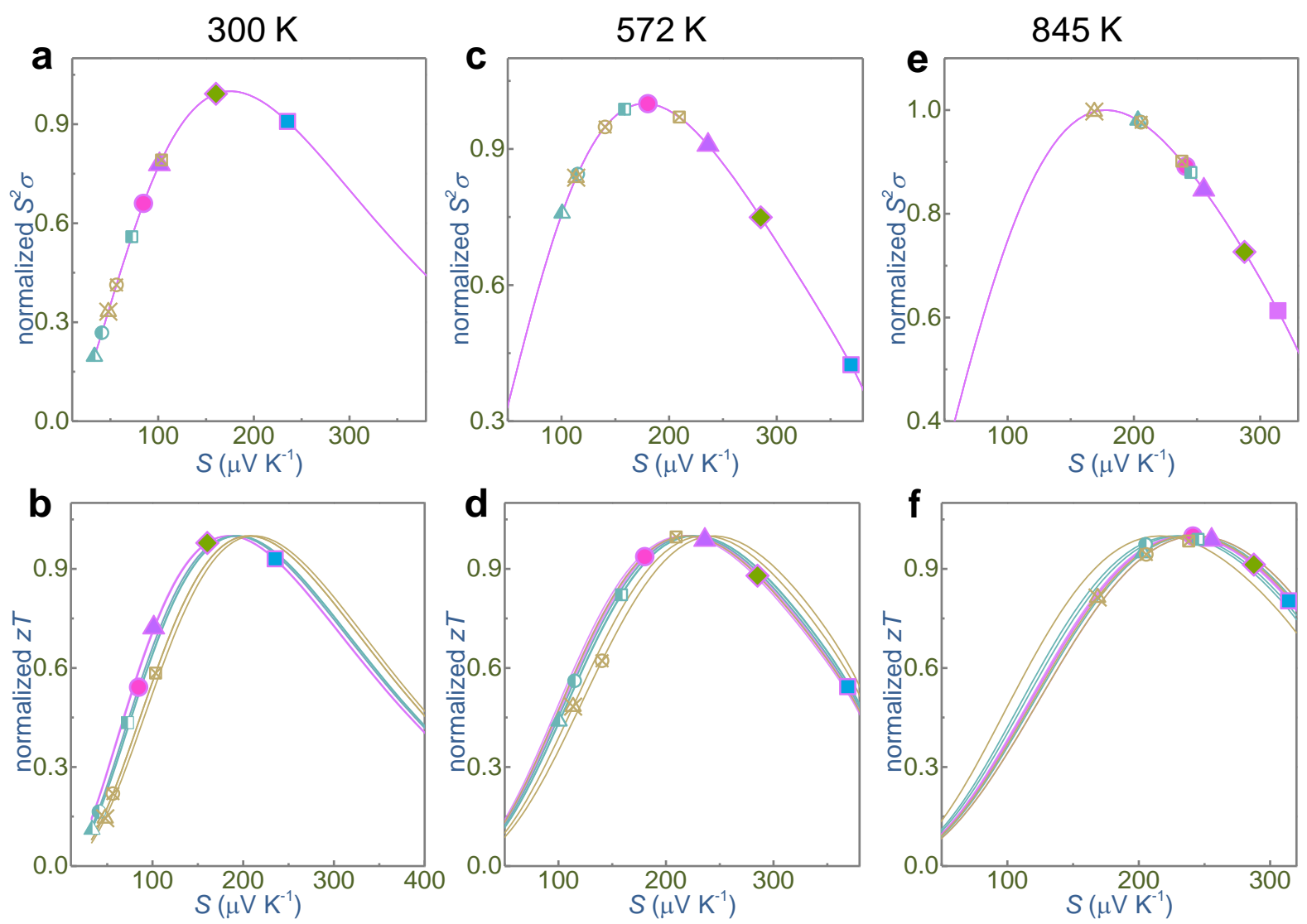

Figure S4 Calculated normalized $S^{2} \sigma$ and normalized $z T$ as a function Seebeck coefficient $(S)$ compared with the data points against $S$ at (a, b) $300 \mathrm{~K}$, (c, d) $572 \mathrm{~K}$, and (e, f) $845 \mathrm{~K}$. 


\section{Hall measurement results}
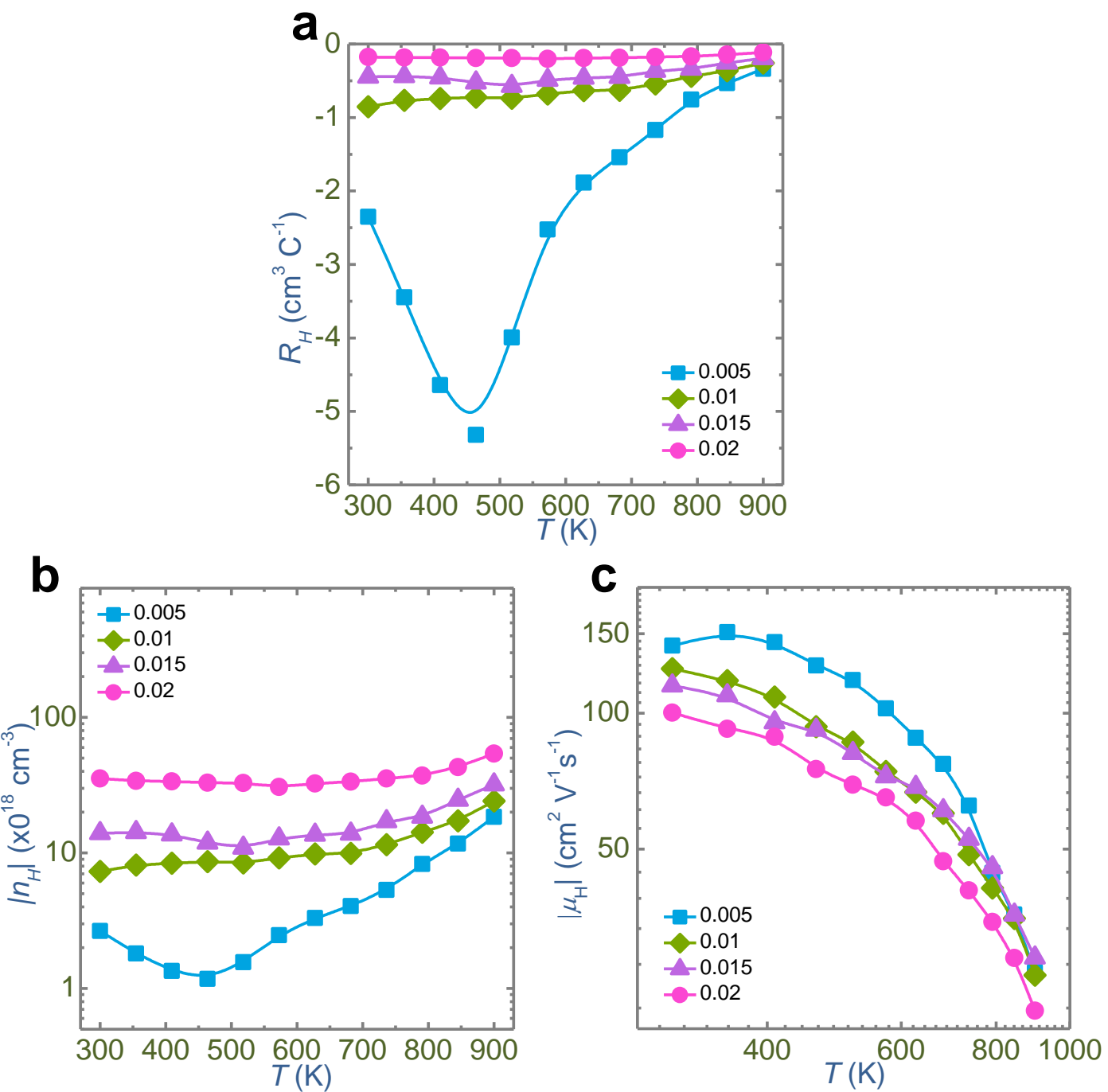

Figure S5 (a) Measured Hall coefficient $\left(R_{\mathrm{H}}\right)$, (b) Hall carrier concentration $\left(n_{\mathrm{H}}\right)$, and (c) Hall carrier mobility $\left(\mu_{\mathrm{H}}\right)$. 


\section{Calculation of $\eta, m_{\mathrm{d}}^{*}$, and $E_{\text {def }}$ of $P b_{1-x} B_{x} S e$}

Figure S6 plots the calculated $\eta, m_{\mathrm{d}}^{*}$, and $E_{\mathrm{def}}$ of $\mathrm{Pb}_{1-\mathrm{x}} \mathrm{Bi} \mathrm{x}_{\mathrm{x}} \mathrm{Se}$. In Figure S6a, with increasing Bi content in $\mathrm{Pb}_{1-\mathrm{x}} \mathrm{Bi}_{\mathrm{x}} \mathrm{Se}, \eta$ keeps increasing. Since $\mathrm{Bi}$ has a valence of $3+$ when substituting $\mathrm{Pb}$ in the lattice of PbSe, Bi server as the donors to shift Fermi level into the conduction band. In Figure S6b, the calculated $m_{\mathrm{d}}^{*}$ for different samples displays an increasing trend with increasing temperature, agreeing with the variation of temperature-dependent $m_{\mathrm{d}}^{*}$ for $\mathrm{PbSe}$-based materials ${ }^{48}$. In Figure S6c, the calculated $E_{\text {def }}$ decreases with increasing temperature for $\mathrm{Pb}_{1-\mathrm{x}} \mathrm{Bi}_{\mathrm{x}} \mathrm{Se}$.
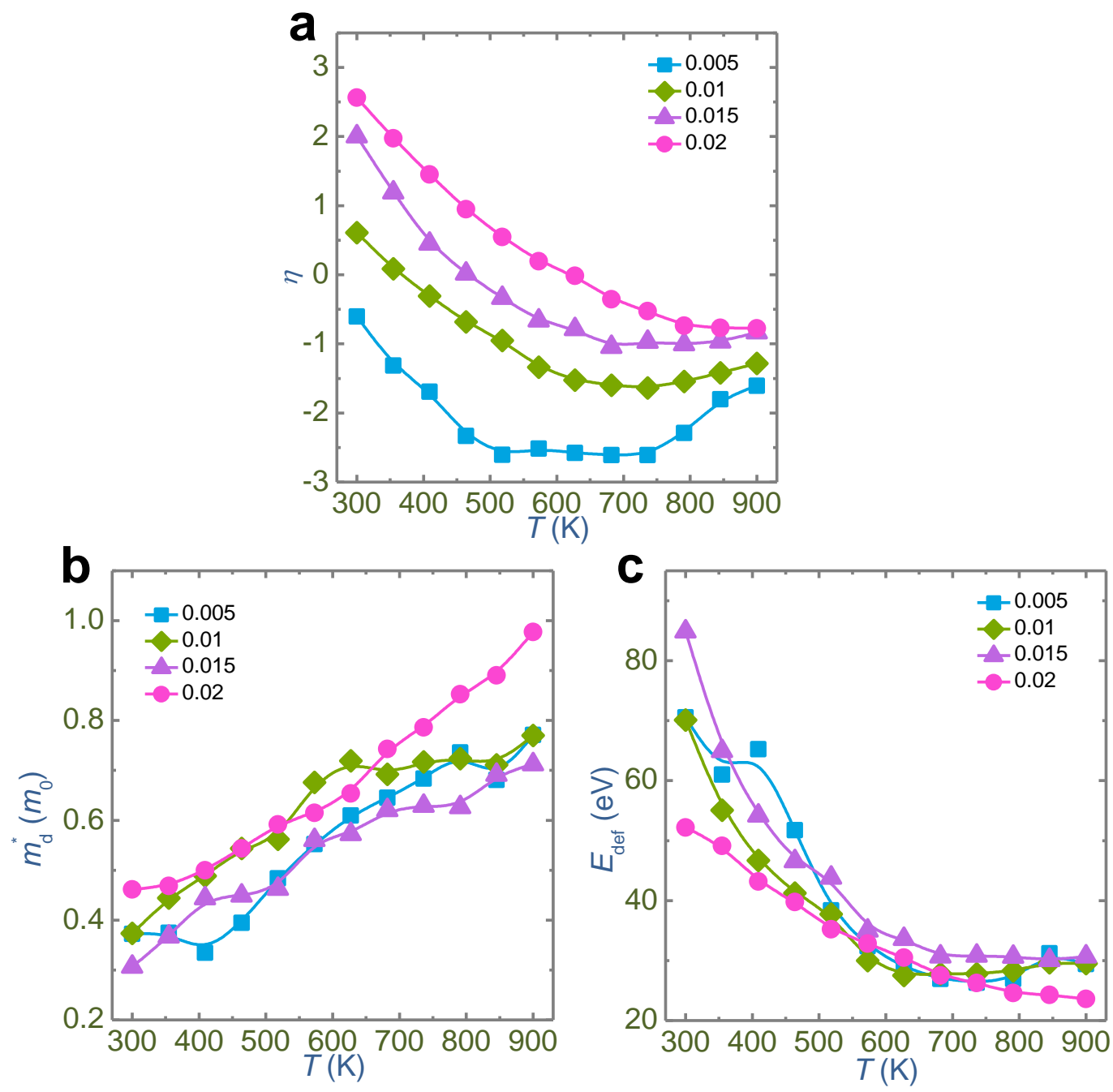

Figure S6 Calculated (a) $\eta$, (b) $m_{\mathrm{d}}^{*}$, and (c) $E_{\text {def }}$ of $\mathrm{Pb}_{1-\mathrm{x}} \mathrm{Bi}_{\mathrm{x}} \mathrm{Se}$. 
Figure S7 and Figure S8 shows the calculated $\eta, m_{\mathrm{d}}^{*}$, and $E_{\mathrm{def}}$ of $p$-type $\mathrm{Pb}_{1-\mathrm{x}} \mathrm{Na}_{\mathrm{x}} \mathrm{Se}^{46}$ and $n$ type $\mathrm{Pb}_{1.002} \mathrm{Se}_{1-\mathrm{x}} \mathrm{Br}_{\mathrm{x}}{ }^{48}$, respectively.

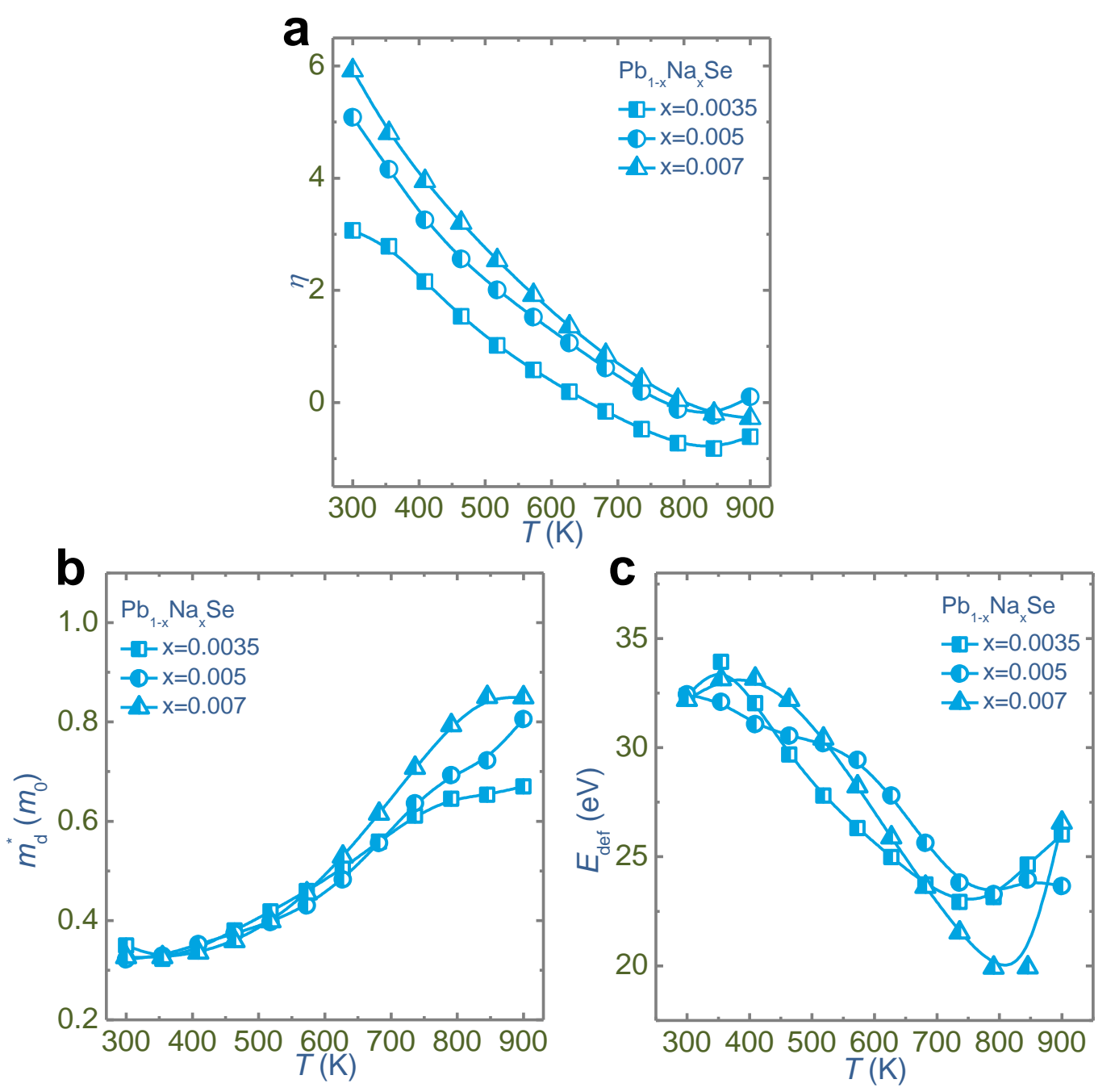

Figure S7 Calculated (a) $\eta$, (b) $m_{\mathrm{d}}^{*}$, and (c) $E_{\text {def }}$ of $p$-type $\mathrm{Pb}_{1-\mathrm{x}} \mathrm{Na}_{\mathrm{x}} \mathrm{Se}^{46}$ 

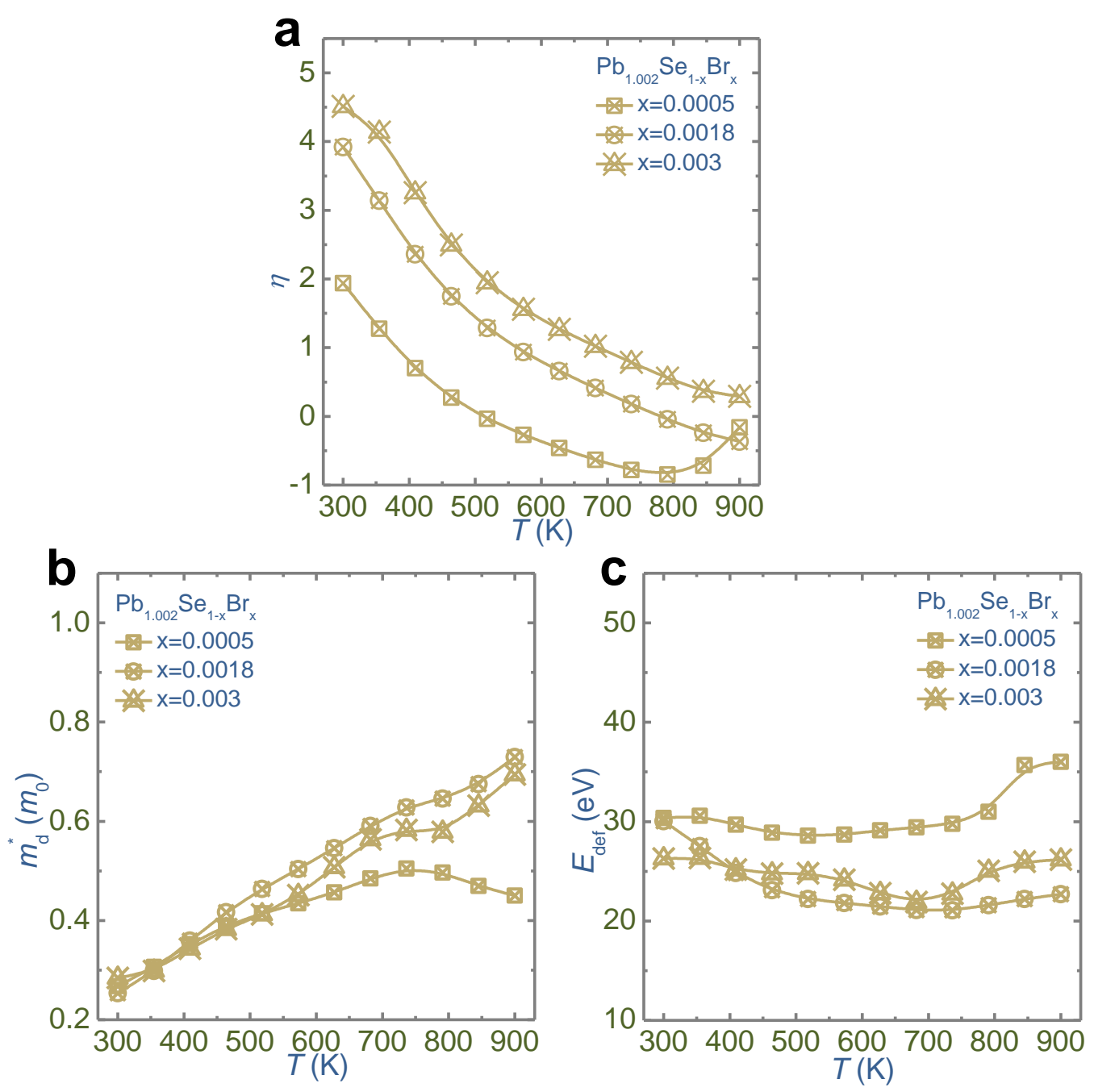

Figure S8 Calculated (a) $\eta$, (b) $m_{\mathrm{d}}^{*}$, and (c) $E_{\text {def }}$ of $n$-type $\mathrm{Pb}_{1.002} \mathrm{Se}_{1-\mathrm{x}} \mathrm{Br}_{\mathrm{x} .}{ }^{48}$ 


\section{Three-dimensional plots compared with the experimental data points}

Figure $S 9$ and Figure $S 10$ present calculated curves of $S, \mu_{H}, S^{2} \sigma$, and $z T$ as functions of $n_{H}$ and temperature, compared with the corresponding data points for $p$-type $\mathrm{Pb}_{1-\mathrm{x}} \mathrm{Na}_{\mathrm{x}} \mathrm{Se}^{46}$ and $n$-type $\mathrm{Pb}_{1.002} \mathrm{Se}_{1-\mathrm{x}} \mathrm{Br}_{\mathrm{x}}{ }^{48}$ respectively. The curves are calculated using the corresponding parameters shown in Figure S7 and Figure S8. All the data points well fit with the plots, verifying the calculation of these parameters.
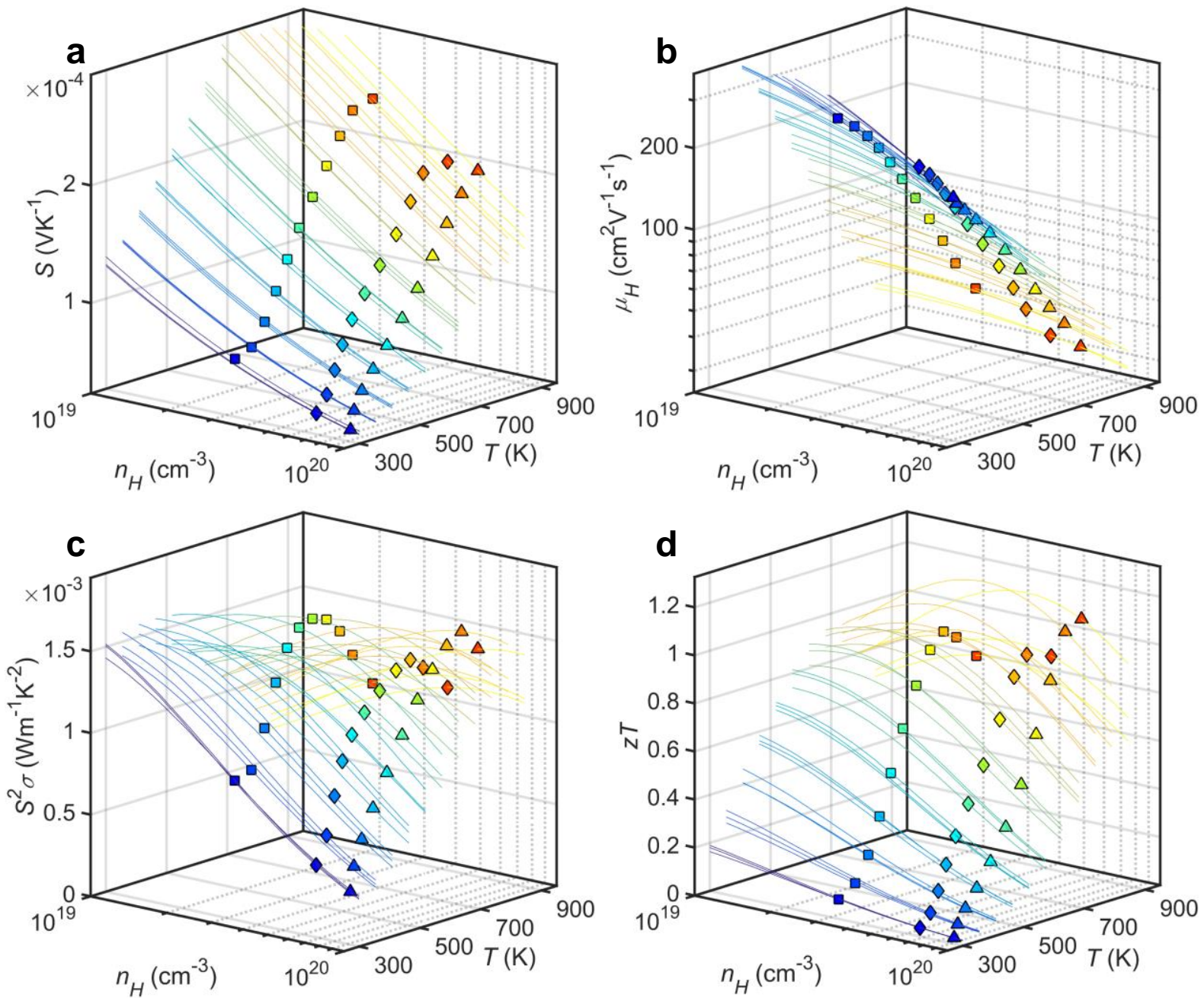

Figure S9 Calculated curves of (a) $S$, (b) $\mu_{H}$, (c) $S^{2} \sigma$, and (d) $z T$ as functions of $n_{H}$ and temperature, compared with the corresponding data points for $p$-type $\mathrm{Pb}_{1-\mathrm{x}} \mathrm{Na}_{\mathrm{x}} \mathrm{Se}^{46}$ 

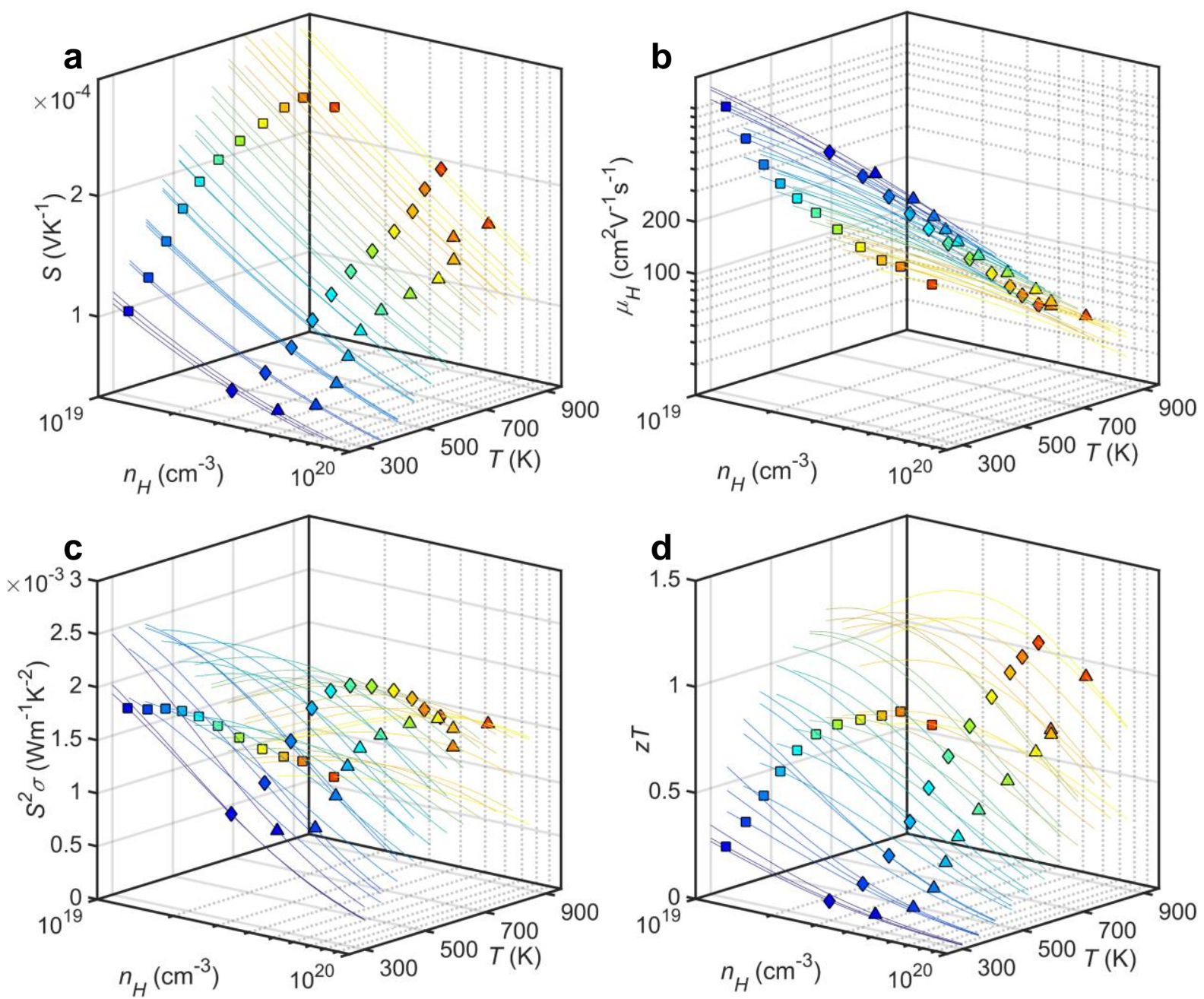

Figure S10 Calculated curves of (a) $S$, (b) $\mu_{H}$, (c) $S^{2} \sigma$, and (d) $z T$ as functions of $n_{H}$ and temperature, compared with the corresponding data points for $n$-type $\mathrm{Pb}_{1.002} \mathrm{Se}_{1-\mathrm{x}} \mathrm{Br}_{\mathrm{x} .}{ }^{48}$ 


\section{Calculated curves of $S$ and $\mu_{\mathrm{H}}$ as a function of $n_{\mathrm{H}}$}

Figure $\mathrm{S} 11$ shows the calculated curves of $\left|\mu_{\mathrm{H}}\right|$ as a function of $\left|n_{\mathrm{H}}\right|$ at three typical temperatures of 300,572 , and $845 \mathrm{~K}$. The data points from this study and from the literature $p$-type $\mathrm{Pb}_{1-}$ ${ }_{x} \mathrm{Na}_{\mathrm{x}} \mathrm{Se}^{46}$ and $n$-type $\mathrm{Pb}_{1.002} \mathrm{Se}_{1-\mathrm{x}} \mathrm{Br}_{\mathrm{x}}{ }^{48}$ are analyzed in these plots.
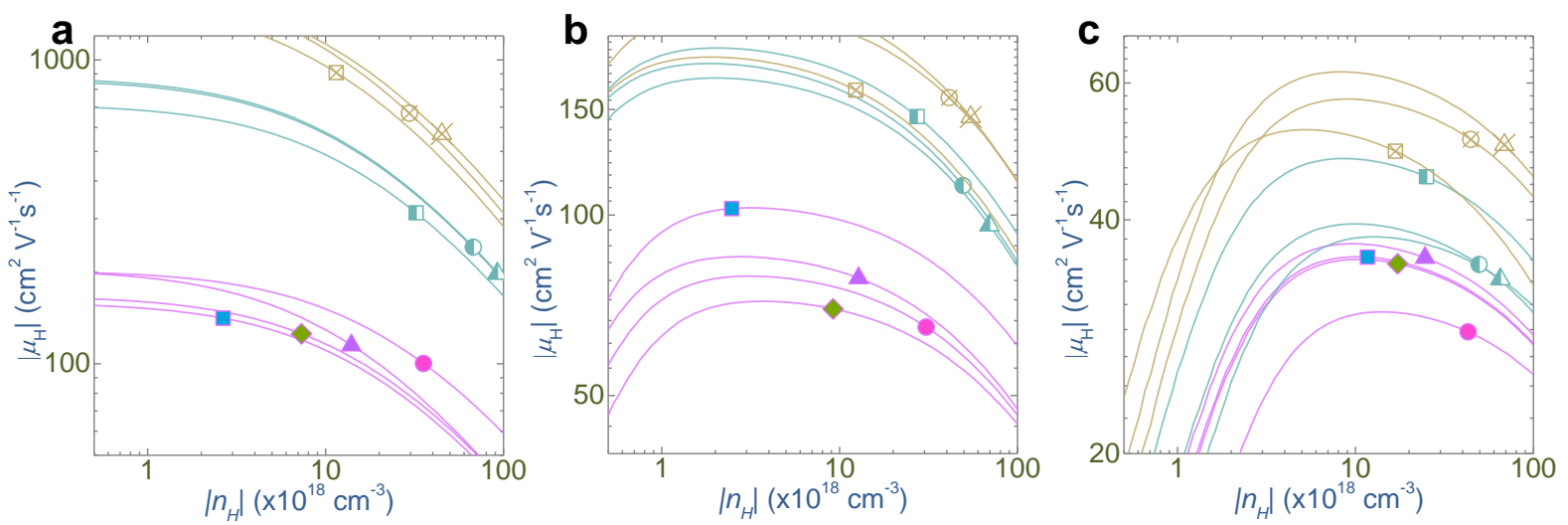

Figure S11 Calculated curves of $\left|\mu_{\mathrm{H}}\right|$ as a function of $\left|n_{\mathrm{H}}\right|$ at (a) 300 , (b) 572 , and (c) $845 \mathrm{~K}$. The corresponding data points of as-prepared $\mathrm{Pb}_{1-\mathrm{x}} \mathrm{Bi} \mathrm{i}_{\mathrm{x}} \mathrm{Se}$, p-type $\mathrm{Pb}_{1-\mathrm{x}} \mathrm{Na}_{\mathrm{x}} \mathrm{Se}^{46}$ and $n$-type $\mathrm{Pb}_{1.002} \mathrm{Se}_{1-\mathrm{x}} \mathrm{Br}_{\mathrm{x}}{ }^{48}$ are included in these plots as well. 


\section{References}

(1) Dismukes, J. P.; Ekstrom, L.; Steigmeier, E. F.; Kudman, I.; Beers, D. S. Thermal and electrical properties of heavily doped Ge-Si alloys up to $1300^{\circ}$ K. J. Appl. Phys. 1964, 35, 28992907.

(2) Joshi, G.; Lee, H.; Lan, Y.; Wang, X.; Zhu, G.; Wang, D.; Gould, R. W.; Cuff, D. C.; Tang, M. Y.; Dresselhaus, M. S.; Chen, G.; Ren, Z. Enhanced thermoelectric figure-of-merit in nanostructured $p$-type silicon germanium bulk alloys. Nano Lett. 2008, 8, 4670-4674.

(3) Wang, X. W.; Lee, H.; Lan, Y. C.; Zhu, G. H.; Joshi, G.; Wang, D. Z.; Yang, J.; Muto, A. J.; Tang, M. Y.; Klatsky, J.; Song, S.; Dresselhaus, M. S.; Chen, G.; Ren, Z. F. Enhanced thermoelectric figure of merit in nanostructured n-type silicon germanium bulk alloy. Appl. Phys. Lett. 2008, 93, 193121.

(4) Tang, Y.; Gibbs, Z. M.; Agapito, L. A.; Li, G.; Kim, H.-S.; Nardelli, M. B.; Curtarolo, S.; Snyder, G. J. Convergence of multi-valley bands as the electronic origin of high thermoelectric performance in $\mathrm{CoSb}_{3}$ skutterudites. Nat. Mater. 2015, 14, 1223-1228.

(5) Tang, Y.; Qiu, Y.; Xi, L.; Shi, X.; Zhang, W.; Chen, L.; Tseng, S.-M.; Chen, S.-w.; Snyder, G. J. Phase diagram of In-Co-Sb system and thermoelectric properties of in-containing skutterudites. Energy Environ. Sci. 2014, 7, 812-819.

(6) Tang, Y.; Hanus, R.; Chen, S.-w.; Snyder, G. J. Solubility design leading to high figure of merit in low-cost Ce-CoSb3 skutterudites. Nat. Commun. 2015, 6, 7584.

(7) Grebenkemper, J. H.; Hu, Y.; Barrett, D.; Gogna, P.; Huang, C.-K.; Bux, S. K.; Kauzlarich, S. M. High temperature thermoelectric properties of $\mathrm{Yb}_{14} \mathrm{MnSb}_{11}$ prepared from reaction of $\mathrm{MnSb}$ with the elements. Chem. Mater. 2015, 27, 5791-5798.

(8) Zhao, W.; Liu, Z.; Wei, P.; Zhang, Q.; Zhu, W.; Su, X.; Tang, X.; Yang, J.; Liu, Y.; Shi, J.; Chao, Y.; Lin, S.; Pei, Y. Magnetoelectric interaction and transport behaviours in magnetic nanocomposite thermoelectric materials. Nat. Nano. 2016, 12, 55-60.

(9) Zhao, W.; Liu, Z.; Sun, Z.; Zhang, Q.; Wei, P.; Mu, X.; Zhou, H.; Li, C.; Ma, S.; He, D.; Ji, P.; Zhu, W.; Nie, X.; Su, X.; Tang, X.; Shen, B.; Dong, X.; Yang, J.; Liu, Y.; Shi, J. Superparamagnetic enhancement of thermoelectric performance. Nature 2017, 549, 247-251.

(10) Meng, X.; Liu, Z.; Cui, B.; Qin, D.; Geng, H.; Cai, W.; Fu, L.; He, J.; Ren, Z.; Sui, J. Grain boundary engineering for achieving high thermoelectric performance in n-type Skutterudites. Adv. Energy Mater. 2017, 7, 1602582.

(11) Li, H.; Su, X.; Tang, X.; Zhang, Q.; Uher, C.; Snyder, G. J.; Aydemir, U. Grain boundary engineering with nano-scale InSb producing high performance $\mathrm{In}_{\mathrm{x}} \mathrm{Ce}_{\mathrm{y}} \mathrm{Co}_{4} \mathrm{Sb}_{12+\mathrm{z}}$ skutterudite thermoelectrics. J. Materiomics 2017, 3, 273-279.

(12) Duan, B.; Zhai, P. C.; Liu, L. S.; Zhang, Q. J. Effects of Se substitution on the thermoelectric performance of n-type $\mathrm{Co}_{4} \mathrm{Sb}_{11.3} \mathrm{Te}_{0.7-\mathrm{x}} \mathrm{Se}_{\mathrm{x}}$ skutterudites. Mater. Res. Bull. 2012, 47, 1670-1673.

(13) Shi, X.; Yang, J.; Salvador, J. R.; Chi, M.; Cho, J. Y.; Wang, H.; Bai, S.; Yang, J.; Zhang, W.; Chen, L. Multiple-filled Skutterudites: high thermoelectric figure of merit through separately optimizing electrical and thermal transports. J. Am. Chem. Soc. 2011, 133, 78377846.

(14) Fu, C.; Zhu, T.; Liu, Y.; Xie, H.; Zhao, X. Band Engineering of high performance ptype $\mathrm{FeNbSb}$ based half-Heusler thermoelectric materials for figure of merit $Z T>1$. Energy Environ. Sci. 2015, 8, 216-220. 
(15) Fu, C.; Zhu, T.; Pei, Y.; Xie, H.; Wang, H.; Snyder, G. J.; Liu, Y.; Liu, Y.; Zhao, X. High band degeneracy contributes to high thermoelectric performance in p-type half-Heusler compounds. Adv. Energy Mater. 2014, 4, 1400600.

(16) Toberer, E. S.; Cox, C. A.; Brown, S. R.; Ikeda, T.; May, A. F.; Kauzlarich, S. M.; Snyder, G. J. Traversing the metal-insulator transition in a zintl phase: Rational enhancement of thermoelectric efficiency in $\mathrm{Yb}_{14} \mathrm{Mn}_{1-\mathrm{x}} \mathrm{Al}_{\mathrm{x}} \mathrm{Sb}_{11}$. Adv. Funct. Mater. 2008, 18, 2795-2800.

(17) Roychowdhury, S.; Panigrahi, R.; Perumal, S.; Biswas, K. Ultrahigh thermoelectric figure of merit and enhanced mechanical stability of $p$-type $\mathrm{AgSb}_{1-\mathrm{x}} \mathrm{Zn}_{\mathrm{x}} \mathrm{Te}_{2}$. ACS Energy Lett. 2017, 2, 349-356.

(18) He, R.; Kraemer, D.; Mao, J.; Zeng, L.; Jie, Q.; Lan, Y.; Li, C.; Shuai, J.; Kim, H. S.; Liu, Y.; Broido, D.; Chu, C.-W.; Chen, G.; Ren, Z. Achieving high power factor and output power density in p-type half-Heuslers $\mathrm{Nb}_{1-\mathrm{x}} \mathrm{Ti}_{\mathrm{x}} \mathrm{FeSb}$. Proc. Natl. Acad. Sci. 2016, 113, 1357613581.

(19) Berry, T.; Ouardi, S.; Fecher, G. H.; Balke, B.; Kreiner, G.; Auffermann, G.; Schnelle, W.; Felser, C. Improving thermoelectric performance of TiNiSn by mixing MnNiSb in the halfHeusler structure. Phys. Chem. Chem. Phys. 2017, 19, 1543-1550.

(20) Hong, M.; Chen, Z.-G.; Yang, L.; Chasapis, T. C.; Kang, S. D.; Zou, Y.; Auchterlonie, G. J.; Kanatzidis, M. G.; Snyder, G. J.; Zou, J. Enhancing the thermoelectric performance of $\mathrm{SnSe}_{1-\mathrm{x}} \mathrm{Te}_{\mathrm{x}}$ nanoplates through band engineering. J. Mater. Chem. A 2017, 5, 10713-10721.

(21) Sassi, S.; Candolfi, C.; Vaney, J. B.; Ohorodniichuk, V.; Masschelein, P.; Dauscher, A.; Lenoir, B. Assessment of the thermoelectric performance of polycrystalline p-type SnSe. Appl. Phys. Lett. 2014, 104, 212105.

(22) Li, Y.; Shi, X.; Ren, D.; Chen, J.; Chen, L. Investigation of the anisotropic thermoelectric properties of oriented polycrystalline SnSe. Energies 2015, 8, 6275-6285.

(23) Zhang, Q.; Chere, E. K.; Sun, J.; Cao, F.; Dahal, K.; Chen, S.; Chen, G.; Ren, Z. Studies on thermoelectric properties of n-type polycrystalline $\mathrm{SnSe}_{1-\mathrm{x}} \mathrm{S}_{\mathrm{x}}$ by iodine doping. Adv. Energy Mater. 2015, 5, 1500360.

(24) Chen, C. L.; Wang, H.; Chen, Y. Y.; Day, T.; Snyder, G. J. Thermoelectric properties of $p$-type polycrystalline SnSe doped with Ag. J. Mater. Chem. A 2014, 2, 11171-11176.

(25) Wei, T.-R.; Tan, G.; Zhang, X.; Wu, C.-F.; Li, J.-F.; Dravid, V. P.; Snyder, G. J.; Kanatzidis, M. G. Distinct impact of alkali-ion doping on electrical transport properties of thermoelectric p-type polycrystalline SnSe. J. Am. Chem. Soc. 2016, 138, 8875-8882.

(26) Wei, T.-R.; Wu, C.-F.; Zhang, X.; Tan, Q.; Sun, L.; Pan, Y.; Li, J.-F. Thermoelectric transport properties of pristine and Na-doped $\mathrm{SnSe}_{1-\mathrm{x}} \mathrm{Te}_{\mathrm{x}}$ polycrystals. Phys. Chem. Chem. Phys. 2015, 17, 30102-30109.

(27) Zhao, L.-D.; Tan, G.; Hao, S.; He, J.; Pei, Y.; Chi, H.; Wang, H.; Gong, S.; Xu, H.; Dravid, V. P.; Uher, C.; Snyder, G. J.; Wolverton, C.; Kanatzidis, M. G. Ultrahigh power factor and thermoelectric performance in hole-doped single-crystal SnSe. Science 2016, 351, 141144.

(28) Zhao, L.; Lo, S. H.; Zhang, Y.; Sun, H.; Tan, G.; Uher, C.; Wolverton, C.; Dravid, V. P.; Kanatzidis, M. G. Ultralow thermal conductivity and high thermoelectric figure of merit in SnSe crystals. Nature 2014, 508, 373-377.

(29) Peng, K.; Lu, X.; Zhan, H.; Hui, S.; Tang, X.; Wang, G.; Dai, J.; Uher, C.; Wang, G.; Zhou, X. Broad temperature plateau for high ZTs in heavily doped p-type SnSe single crystals. Energy Environ. Sci. 2015, 9, 454-460.

(30) Zhao, L.-D.; Zhang, X.; Wu, H.; Tan, G.; Pei, Y.; Xiao, Y.; Chang, C.; Wu, D.; Chi, H.; Zheng, L.; Gong, S.; Uher, C.; He, J.; Kanatzidis, M. G. Enhanced thermoelectric properties 
in the counter-doped SnTe system with strained endotaxial SrTe. J. Am. Chem. Soc. 2016, 138, 2366-2373.

(31) Tan, G.; Shi, F.; Doak, J. W.; Sun, H.; Zhao, L.-D.; Wang, P.; Uher, C.; Wolverton, C.; Dravid, V. P.; Kanatzidis, M. G. Extraordinary role of $\mathrm{Hg}$ in enhancing the thermoelectric performance of p-type SnTe. Energy Environ. Sci. 2015, 8, 267-277.

(32) Li, W.; Zheng, L.; Ge, B.; Lin, S.; Zhang, X.; Chen, Z.; Chang, Y.; Pei, Y. Promoting $\mathrm{SnTe}$ as an eco-friendly solution for $p$-PbTe thermoelectric via band convergence and interstitial defects. Adv. Mater. 2017, 29, 1605887.

(33) Tan, G.; Zhao, L.-D.; Shi, F.; Doak, J. W.; Lo, S.-H.; Sun, H.; Wolverton, C.; Dravid, V. P.; Uher, C.; Kanatzidis, M. G. High thermoelectric performance of $p$-Type SnTe via a synergistic band engineering and nanostructuring approach. J. Am. Chem. Soc. 2014, 136, 7006-7017.

(34) Tan, G.; Zeier, W. G.; Shi, F.; Wang, P.; Snyder, G. J.; Dravid, V. P.; Kanatzidis, M. G. High thermoelectric performance $\mathrm{SnTe}-\mathrm{In}_{2} \mathrm{Te}_{3}$ solid solutions enabled by resonant levels and strong vacancy phonon scattering. Chem. Mater. 2015, 27, 7801-7811.

(35) Tan, G.; Shi, F.; Hao, S.; Chi, H.; Zhao, L.-D.; Uher, C.; Wolverton, C.; Dravid, V. P.; Kanatzidis, M. G. Codoping in SnTe: enhancement of thermoelectric performance through synergy of resonance levels and band convergence. J. Am. Chem. Soc. 2015, 137, 5100-5112.

(36) Tan, G.; Shi, F.; Hao, S.; Chi, H.; Bailey, T. P.; Zhao, L.-D.; Uher, C.; Wolverton, C.; Dravid, V. P.; Kanatzidis, M. G. Valence band modification and high thermoelectric performance in SnTe heavily alloyed with MnTe. J. Am. Chem. Soc. 2015, 137, 11507-11516.

(37) Tan, G.; Hao, S.; Hanus, R. C.; Zhang, X.; Anand, S.; Bailey, T. P.; Rettie, A. J. E.; Su, X.; Uher, C.; Dravid, V. P.; Snyder, G. J.; Wolverton, C.; Kanatzidis, M. G. High thermoelectric performance in $\mathrm{SnTe}-\mathrm{AgSbTe}_{2}$ alloys from lattice softening, giant phononvacancy scattering, and valence band convergence. ACS Energy Lett. 2018, 3, 705-712.

(38) Zhou, M.; Gibbs, Z. M.; Wang, H.; Han, Y.; Xin, C.; Li, L.; Snyder, G. J. Optimization of thermoelectric efficiency in SnTe: the case for the light band. Phys. Chem. Chem. Phys. 2014, 16, 20741-20748.

(39) Zheng, L.; Li, W.; Lin, S.; Li, J.; Chen, Z.; Pei, Y. Interstitial defects improving thermoelectric SnTe in addition to band convergence. ACS Energy Lett. 2017, 563-568.

(40) Zhang, Q.; Liao, B.; Lan, Y.; Lukas, K.; Liu, W.; Esfarjani, K.; Opeil, C.; Broido, D.; Chen, G.; Ren, Z. High thermoelectric performance by resonant dopant indium in nanostructured SnTe. Proc. Natl. Acad. Sci. USA 2013, 110, 13261-13266.

(41) Pei, Y.; Zheng, L.; Li, W.; Lin, S.; Chen, Z.; Wang, Y.; Xu, X.; Yu, H.; Chen, Y.; Ge, B. Interstitial point defect scattering contributing to high thermoelectric performance in SnTe. Adv. Electron. Mater. 2016, 2, 1600019.

(42) Li, W.; Chen, Z.; Lin, S.; Chang, Y.; Ge, B.; Chen, Y.; Pei, Y. Band and scattering tuning for high performance thermoelectric $\mathrm{Sn}_{1-\mathrm{x}} \mathrm{Mn}_{\mathrm{x}} \mathrm{Te}$ alloys. J. Materiomics 2015, 1, 307315.

(43) Banik, A.; Shenoy, U. S.; Anand, S.; Waghmare, U. V.; Biswas, K. Mg alloying in SnTe facilitates valence band convergence and optimizes thermoelectric properties. Chem. Mater. 2015, 27, 581-587.

(44) Pei, Y.; Shi, X.; LaLonde, A.; Wang, H.; Chen, L.; Snyder, G. J. Convergence of electronic bands for high performance bulk thermoelectrics. Nature 2011, 473, 66-69.

(45) Yamini, S. A.; Mitchell, D. R. G.; Gibbs, Z. M.; Santos, R.; Patterson, V.; Li, S.; Pei, Y. Z.; Dou, S. X.; Jeffrey Snyder, G. Heterogeneous distribution of sodium for high 
thermoelectric performance of p-type multiphase lead-chalcogenides. Adv. Energy Mater. 2015, 5, 1501047.

(46) Wang, H.; Pei, Y.; LaLonde, A. D.; Snyder, G. J. Heavily doped $p$-type PbSe with high thermoelectric performance: an alternative for PbTe. Adv. Mater. 2011, 23, 1366-1370.

(47) Wang, H.; Gibbs, Z. M.; Takagiwa, Y.; Snyder, G. J. Tuning bands of PbSe for better thermoelectric efficiency. Energy Environ. Sci. 2014, 7, 804-811.

(48) Wang, H.; Pei, Y.; LaLonde, A. D.; Snyder, G. J. Weak electron-phonon coupling contributing to high thermoelectric performance in n-type PbSe. Proc. Natl. Acad. Sci. USA 2012, 109, 9705-9709.

(49) Wang, H.; Schechtel, E.; Pei, Y.; Snyder, G. J. High thermoelectric efficiency of $n$-type PbS. Adv. Energy Mater. 2013, 3, 488-495.

(50) Lee, Y.; Lo, S.-H.; Chen, C.; Sun, H.; Chung, D.-Y.; Chasapis, T. C.; Uher, C.; Dravid, V. P.; Kanatzidis, M. G. Contrasting role of antimony and bismuth dopants on the thermoelectric performance of lead selenide. Nat. Commun. 2014, 5, 4640.

(51) Biswas, K.; He, J.; Blum, I. D.; Wu, C. I.; Hogan, T. P.; Seidman, D. N.; Dravid, V. P.; Kanatzidis, M. G. High-performance bulk thermoelectrics with all-scale hierarchical architectures. Nature 2012, 489, 414-418.

(52) Wu, H. J.; Zhao, L. D.; Zheng, F. S.; Wu, D.; Pei, Y. L.; Tong, X.; Kanatzidis, M. G.; $\mathrm{He}$, J. Q. Broad temperature plateau for thermoelectric figure of merit $Z T>2$ in phase-separated $\mathrm{PbTe}_{0.7} \mathrm{~S}_{0.3}$. Nat. Commun. 2014, 5, 4515.

(53) Chen, Z.; Jian, Z.; Li, W.; Chang, Y.; Ge, B.; Hanus, R.; Yang, J.; Chen, Y.; Huang, M.; Snyder, G. J.; Pei, Y. Lattice dislocations enhancing thermoelectric PbTe in addition to band convergence. Adv. Mater. 2017, 29, 1606768.

(54) Chen, Z.; Ge, B.; Li, W.; Lin, S.; Shen, J.; Chang, Y.; Hanus, R.; Snyder, G. J.; Pei, Y. Vacancy-induced dislocations within grains for high-performance PbSe thermoelectrics. Nat. Commun. 2017, 8, 13828.

(55) Zhang, Q.; Cao, F.; Lukas, K.; Liu, W.; Esfarjani, K.; Opeil, C.; Broido, D.; Parker, D.; Singh, D. J.; Chen, G.; Ren, Z. Study of the thermoelectric properties of lead selenide doped with boron, gallium, indium, or thallium. J. Am. Chem. Soc. 2012, 134, 17731-17738.

(56) Zhang, Q.; Chere, E. K.; McEnaney, K.; Yao, M.; Cao, F.; Ni, Y.; Chen, S.; Opeil, C.; Chen, G.; Ren, Z. Enhancement of thermoelectric performance of $n$-type PbSe by $\mathrm{Cr}$ doping with optimized carrier concentration. Adv. Energy Mater. 2015, 5, 1401977.

(57) LaLonde, A. D.; Pei, Y.; Snyder, G. J. Reevaluation of $\mathrm{PbTe}_{1-\mathrm{x}} \mathrm{I}_{\mathrm{x}}$ as high performance n-type thermoelectric material. Energy Environ. Sci. 2011, 4, 2090-2096.

(58) Zhao, L.; Lo, S. H.; He, J.; Li, H.; Biswas, K.; Androulakis, J.; Wu, C.-I.; Hogan, T. P.; Chung, D.-Y.; Dravid, V. P.; Kanatzidis, M. G. High performance thermoelectrics from earthabundant materials: enhanced figure of merit in $\mathrm{PbS}$ by second phase nanostructures. J. Am. Chem. Soc. 2011, 133, 20476-20487.

(59) Zhao, L. D.; Wu, H. J.; Hao, S. Q.; Wu, C. I.; Zhou, X. Y.; Biswas, K.; He, J. Q.; Hogan, T. P.; Uher, C.; Wolverton, C.; Dravid, V. P.; Kanatzidis, M. G. All-scale hierarchical thermoelectrics: MgTe in PbTe facilitates valence band convergence and suppresses bipolar thermal transport for high performance. Energy Environ. Sci. 2013, 6, 3346-3355.

(60) Zhao, L.-D.; Hao, S.; Lo, S.-H.; Wu, C.-I.; Zhou, X.; Lee, Y.; Li, H.; Biswas, K.; Hogan, T. P.; Uher, C.; Wolverton, C.; Dravid, V. P.; Kanatzidis, M. G. High thermoelectric performance via hierarchical compositionally alloyed nanostructures. J. Am. Chem. Soc. 2013, 135, 7364-7370. 
(61) Koenig, J.; Winkler, M.; Dankwort, T.; Hansen, A. L.; Pernau, H. F.; Duppel, V.; Jaegle, M.; Bartholome, K.; Kienle, L.; Bensch, W. Thermoelectric efficiency of (1 $\mathrm{x})(\mathrm{GeTe}) \mathrm{x}\left(\mathrm{Bi}_{2} \mathrm{Se}_{0.2} \mathrm{Te}_{2.8}\right)$ and implementation into highly performing thermoelectric power generators. Dalton Trans. 2015, 44, 2835-2843.

(62) Perumal, S.; Roychowdhury, S.; Negi, D. S.; Datta, R.; Biswas, K. High thermoelectric performance and enhanced mechanical stability of $p$-type $\mathrm{Ge}_{1-\mathrm{x}} \mathrm{S} b_{\mathrm{x}} \mathrm{Te}$. Chem. Mater. 2015, 27, 7171-7178.

(63) Li, J.; Chen, Z.; Zhang, X.; Sun, Y.; Yang, J.; Pei, Y. Electronic origin of the high thermoelectric performance of GeTe among the $p$-type group IV monotellurides. NPG Asia Mater. 2017, 9, e353.

(64) Li, J.; Zhang, X.; Lin, S.; Chen, Z.; Pei, Y. Realizing the high thermoelectric performance of GeTe by Sb-doping and Se-alloying. Chem. Mater. 2016, 29, 605-611.

(65) Samanta, M.; Biswas, K. Low thermal conductivity and high thermoelectric performance in $(\mathrm{GeTe})_{1-2 \mathrm{x}}(\mathrm{GeSe})_{\mathrm{x}}(\mathrm{GeS})_{\mathrm{x}}$ : competition between solid solution and phase separation. J. Am. Chem. Soc. 2017, 139, 9382-9391.

(66) Hong, M.; Wang, Y.; Liu, W.; Matsumura, S.; Wang, H.; Zou, J.; Chen, Z.-G. Arrays of planar vacancies in superior thermoelectric $\mathrm{Ge}_{1-\mathrm{x}-\mathrm{y}} \mathrm{Cd}_{\mathrm{x}} \mathrm{Bi}_{\mathrm{y}} \mathrm{Te}$ with band convergence. $A d v$. Energy Mater. 2018, 8, 1801837.

(67) Williams, J. B.; Morelli, D. T. Understanding the superior thermoelectric performance of Sb precipitated $\mathrm{Ge}_{17} \mathrm{Sb}_{2} \mathrm{Te}_{20}$. J. Mater. Chem. C 2016, 4, 10011-10017.

(68) Hong, M.; Chen, Z.-G.; Yang, L.; Zou, Y.-C.; Dargusch, M. S.; Wang, H.; Zou, J. Realizing $z T$ of 2.3 in $\mathrm{Ge}_{1-\mathrm{x}-\mathrm{y}} \mathrm{Sb}_{\mathrm{x}} \mathrm{In}_{\mathrm{y}} \mathrm{Te}$ via reducing the phase-transition temperature and introducing resonant energy doping. Adv. Mater. 2018, 30, 1705942.

(69) Wu, D.; Zhao, L.; Hao, S.; Jiang, Q.; Zheng, F.; Doak, J. W.; Wu, H.; Chi, H.; Gelbstein, Y.; Uher, C.; Wolverton, C.; Kanatzidis, M.; He, J. Origin of the High Performance in GeTeBased Thermoelectric Materials upon $\mathrm{Bi}_{2} \mathrm{Te}_{3}$ Doping. J. Am. Chem. Soc. 2014, 136, $11412-$ 11419.

(70) Li, J.; Chen, Z.; Zhang, X.; Yu, H.; Wu, Z.; Xie, H.; Chen, Y.; Pei, Y. Simultaneous optimization of carrier concentration and alloy scattering for ultrahigh performance GeTe thermoelectrics. Adv. Sci. 2017, 4, 1700341.

(71) Gelbstein, Y.; Davidow, J.; Girard, S. N.; Chung, D. Y.; Kanatzidis, M. Controlling metallurgical phase separation reactions of the $\mathrm{Ge}_{0.87} \mathrm{~Pb}_{0.13}$ Te alloy for high thermoelectric performance. Adv. Energy Mater. 2013, 3, 815-820.

(72) Zhang, X.; Li, J.; Wang, X.; Chen, Z.; Mao, J.; Chen, Y.; Pei, Y. Vacancy manipulation for thermoelectric enhancements in GeTe alloys. J. Am. Chem. Soc. 2018, 140, 15883-15888.

(73) Gelbstein, Y.; Davidow, J. Highly efficient functional $\mathrm{Ge}_{\mathrm{x}} \mathrm{Pb}_{1-\mathrm{x}} \mathrm{Te}$ based thermoelectric alloys. Phys. Chem. Chem. Phys. 2014, 16, 20120-20126.

(74) Li, J.; Zhang, X.; Chen, Z.; Lin, S.; Li, W.; Shen, J.; Witting, I. T.; Faghaninia, A.; Chen, Y.; Jain, A.; Chen, L.; Snyder, G. J.; Pei, Y. Low-symmetry rhombohedral GeTe thermoelectrics. Joule 2018, 2, 976-987.

(75) Zheng, Z.; Su, X.; Deng, R.; Stoumpos, C.; Xie, H.; Liu, W.; Yan, Y.; Hao, S.; Uher, C.; Wolverton, C.; Kanatzidis, M. G.; Tang, X. Rhombohedral to cubic conversion of GeTe via MnTe alloying leads to ultralow thermal conductivity, electronic band convergence, and high thermoelectric performance. J. Am. Chem. Soc. 2018, 140, 2673-2686.

(76) Wu, L.; Li, X.; Wang, S.; Zhang, T.; Yang, J.; Zhang, W.; Chen, L.; Yang, J. Resonant level-induced high thermoelectric response in indium-doped GeTe. NPG Asia Mater. 2017, 9, e343. 
(77) Davidow, J.; Gelbstein, Y. A comparison between the mechanical and thermoelectric properties of three highly efficient p-type GeTe-rich compositions: TAGS-80, TAGS-85, and $3 \% \mathrm{Bi}_{2} \mathrm{Te}_{3}$-doped $\mathrm{Ge}_{0.87} \mathrm{~Pb}_{0.13}$ Te. J. Electron. Mater. 2013, 42, 1542-1549.

(78) Levin, E. M.; Bud'ko, S. L.; Schmidt-Rohr, K. Enhancement of thermopower of TAGS85 high-performance thermoelectric material by doping with the rare earth Dy. Adv. Funct. Mater. 2012, 22, 2766-2774.

(79) Levin, E. M.; Cook, B. A.; Harringa, J. L.; Bud'ko, S. L.; Venkatasubramanian, R.; Schmidt-Rohr, K. Analysis of Ce- and Yb-doped TAGS-85 materials with enhanced thermoelectric figure of merit. Adv. Funct. Mater. 2011, 21, 441-447.

(80) Liu, W.; Kim, H. S.; Chen, S.; Jie, Q.; Lv, B.; Yao, M.; Ren, Z.; Opeil, C. P.; Wilson, S.; Chu, C.-W.; Ren, Z. n-type thermoelectric material $\mathrm{Mg}_{2} \mathrm{Sn}_{0.75} \mathrm{Ge}_{0.25}$ for high power generation. Proc. Natl. Acad. Sci. USA 2015, 112, 3269-3274.

(81) Liu, W.; Tan, X.; Yin, K.; Liu, H.; Tang, X.; Shi, J.; Zhang, Q.; Uher, C. Convergence of conduction bands as a means of enhancing thermoelectric performance of $n$-type $\mathrm{Mg}_{2} \mathrm{Si}_{1}$ ${ }_{\mathrm{x}} \mathrm{Sn}_{\mathrm{x}}$ solid solutions. Phys. Rev. Lett. 2012, 108, 166601.

(82) Liu, W.; Zhou, J.; Jie, Q.; Li, Y.; Kim, H. S.; Bao, J.; Chen, G.; Ren, Z. New insight into the material parameter $B$ to understand the enhanced thermoelectric performance of $\mathrm{Mg}_{2} \mathrm{Sn}_{1-\mathrm{x}-\mathrm{y}} \mathrm{Ge}_{\mathrm{x}} \mathrm{Sb}_{\mathrm{y}}$. Energy Environ. Sci. 2015, 9, 530-539.

(83) Ning, H.; Mastrorillo, G. D.; Grasso, S.; Du, B.; Mori, T.; Hu, C.; Xu, Y.; Simpson, K.; Maizza, G.; Reece, M. J. Enhanced thermoelectric performance of porous magnesium tin silicide prepared using pressure-less spark plasma sintering. J. Mater. Chem. A 2015, 3, 1742617432.

(84) Yin, K.; Su, X.; Yan, Y.; Uher, C.; Tang, X. In situ nanostructure design leading to a high figure of merit in an eco-friendly stable $\mathrm{Mg}_{2} \mathrm{Si}_{0.30} \mathrm{Sn}_{0.70}$ solid solution. $R S C A d v$. 2016, 6 , 16824-16831.

(85) Zaitsev, V. K.; Fedorov, M. I.; Gurieva, E. A.; Eremin, I. S.; Konstantinov, P. P.; Samunin, A. Y.; Vedernikov, M. V. Highly effective $\mathrm{Mg}_{2} \mathrm{Si}_{1-\mathrm{x}} \mathrm{Sn}_{\mathrm{x}}$ thermoelectrics. Phys. Rev. $B$ 2006, 74, 045207.

(86) Zhang, J.; Song, L.; Pedersen, S. H.; Yin, H.; Hung, L. T.; Iversen, B. B. Discovery of high-performance low-cost $n$-type $\mathrm{Mg}_{3} \mathrm{Sb}_{2}$-based thermoelectric materials with multi-valley conduction bands. Nat. Commun. 2017, 8, 13901.

(87) Liu, Z.; Wang, Y.; Mao, J.; Geng, H.; Shuai, J.; Wang, Y.; He, R.; Cai, W.; Sui, J.; Ren, Z. Lithium doping to enhance thermoelectric performance of MgAgSb with weak electronphonon coupling. Adv. Energy Mater. 2016, 6, 1502269.

(88) Imasato, K.; Kang, S. D.; Snyder, G. J. Exceptional thermoelectric performance in $\mathrm{Mg}_{3} \mathrm{Sb}_{0.6} \mathrm{Bi}_{1.4}$ for low-grade waste heat recovery. Energy Environ. Sci. 2019, 12, 965-971.

(89) Pan, Y.; Qiu, Y.; Witting, I.; Zhang, L.; Fu, C.; Li, J.-W.; Huang, Y.; Sun, F.-H.; He, J.; Snyder, G. J.; Felser, C.; Li, J.-F. Synergistic modulation of mobility and thermal conductivity in $(\mathrm{Bi}, \mathrm{Sb})_{2} \mathrm{Te}_{3}$ towards high thermoelectric performance. Energy Environ. Sci. 2019, 12, 624-630.

(90) Mehta, R. J.; Zhang, Y.; Karthik, C.; Singh, B.; Siegel, R. W.; Borca-Tasciuc, T.; Ramanath, G. A new class of doped nanobulk high figure-of-merit thermoelectrics by scalable bottom-up assembly. Nat. Mater. 2012, 11, 233-240.

(91) Hong, M.; Chen, Z. G.; Yang, L.; Zou, J. Bi $\mathrm{S}_{\mathrm{x}} \mathrm{Sb}_{2-\mathrm{x}} \mathrm{Te}_{3}$ Nanoplates with Enhanced Thermoelectric Performance due to Sufficiently Decoupled Electronic Transport Properties and Strong Wide-Frequency Phonon Scatterings. Nano Energy 2016, 20, 144-155. 
(92) Min, Y.; Roh, J. W.; Yang, H.; Park, M.; Kim, S. I.; Hwang, S.; Lee, S. M.; Lee, K. H.; Jeong, U. Surfactant-free scalable synthesis of $\mathrm{Bi}_{2} \mathrm{Te}_{3}$ and $\mathrm{Bi}_{2} \mathrm{Se}_{3}$ nanoflakes and enhanced thermoelectric properties of their nanocomposites. Adv. Mater. 2013, 25, 1425-9.

(93) Suh, D.; Lee, S.; Mun, H.; Park, S.-H.; Lee, K. H.; Wng Kim, S.; Choi, J.-Y.; Baik, S. Enhanced thermoelectric performance of $\mathrm{Bi}_{0.5} \mathrm{Sb}_{1.5} \mathrm{Te}_{3}$-expanded graphene composites by simultaneous modulation of electronic and thermal carrier transport. Nano Energy 2015, 13, 67-76.

(94) Poudel, B.; Hao, Q.; Ma, Y.; Lan, Y.; Minnich, A.; Yu, B.; Yan, X.; Wang, D.; Muto, A.; Vashaee, D.; Chen, X.; Liu, J.; Dresselhaus, M. S.; Chen, G.; Ren, Z. High-thermoelectric performance of nanostructured bismuth antimony telluride bulk alloys. Science 2008, 320, 634638.

(95) Ma, Y.; Hao, Q.; Poudel, B.; Lan, Y. C.; Yu, B.; Wang, D. Z.; Chen, G.; Ren, Z. F. Enhanced thermoelectric figure-of-merit in p-type nanostructured bismuth antimony tellurium alloys made from elemental chunks. Nano Lett. 2008, 8, 2580-2584.

(96) Prokofieva, L. V.; Pshenay-Severin, D. A.; Konstantinov, P. P.; Shabaldin, A. A. Optimum composition of a $\mathrm{Bi}_{2} \mathrm{Te}_{3-\mathrm{x}} \mathrm{Se}_{\mathrm{x}}$ alloy for the $n$-type leg of a thermoelectric generator. Semiconductors 2009, 43, 973-976.

(97) Wang, S.; Tan, G.; Xie, W.; Zheng, G.; Li, H.; Yang, J.; Tang, X. Enhanced thermoelectric properties of $\mathrm{Bi}_{2}\left(\mathrm{Te}_{1-\mathrm{x}} \mathrm{Se}_{\mathrm{x}}\right)_{3}$-based compounds as $n$-type legs for lowtemperature power generation. J. Mater. Chem. 2012, 22, $20943-20951$.

(98) Xie, W. J.; Tang, X. F.; Yan, Y.; Zhang, Q.; Tritt, T. M. Unique nanostructures and enhanced thermoelectric performance of melt-spun BiSbTe alloys. Appl. Phys. Lett. 2009, 94, 102111.

(99) Liu, W.-S.; Zhang, Q.; Lan, Y.; Chen, S.; Yan, X.; Zhang, Q.; Wang, H.; Wang, D.; Chen, G.; Ren, Z. Thermoelectric property studies on $\mathrm{Cu}$-doped $n$-type $\mathrm{Cu}_{\mathrm{x}} \mathrm{Bi}_{2} \mathrm{Te}_{2.7} \mathrm{Se}_{0.3}$ nanocomposites. Adv. Energy Mater. 2011, 1, 577-587.

(100) Hu, L.; Wu, H.; Zhu, T.; Fu, C.; He, J.; Ying, P.; Zhao, X. Tuning multiscale microstructures to enhance thermoelectric performance of $n$-type bismuth-telluride-based solid solutions. Adv. Energy Mater. 2015, 5, 1500411.

(101) Yan, X.; Poudel, B.; Ma, Y.; Liu, W. S.; Joshi, G.; Wang, H.; Lan, Y.; Wang, D.; Chen, G.; Ren, Z. F. Experimental studies on anisotropic thermoelectric properties and structures of $n$-type $\mathrm{Bi}_{2} \mathrm{Te}_{2.7} \mathrm{Se}_{0.3}$. Nano Lett. 2010, 10, 3373-3378.

(102) Hong, M.; Chen, Z.-G.; Pei, Y.; Yang, L.; Zou, J. Limit of $z T$ enhancement in rocksalt structured chalcogenides by band convergence. Phys. Rev. B 2016, 94, 161201.

(103) Pei, Y.; Gibbs, Z. M.; Balke, B.; Zeier, W. G.; Snyder, G. J. Optimum carrier concentration in $n$-type PbTe thermoelectrics. Adv. Energy Mater. 2014, 4, 1400486.

(104) Liu, W.; Lukas, K. C.; McEnaney, K.; Lee, S.; Zhang, Q.; Opeil, C. P.; Chen, G.; Ren, Z. Studies on the $\mathrm{Bi}_{2} \mathrm{Te}_{3}-\mathrm{Bi}_{2} \mathrm{Se}_{3}-\mathrm{Bi}_{2} \mathrm{~S}_{3}$ system for mid-temperature thermoelectric energy conversion. Energy Environ. Sci. 2013, 6, 552-560.

(105) Callaway, J.; von Baeyer, H. Effect of Point Imperfections on Lattice Thermal Conductivity. Phys. Rev. 1960, 120, 1149-1154.

(106) Bessas, D.; Sergueev, I.; Wille, H. C.; Perßon, J.; Ebling, D.; Hermann, R. P. Lattice Dynamics in $\mathrm{Bi}_{2} \mathrm{Te}_{3}$ and $\mathrm{Sb}_{2} \mathrm{Te}_{3}$ : Te and $\mathrm{Sb}$ Density of Phonon States. Phys. Rev. B 2012, 86, 224301.

(107) Zhu, T. J.; Fu, C. G.; Xie, H. H.; Liu, Y. T.; Feng, B.; Xie, J.; Zhao, X. B. Lattice Thermal Conductivity and Spectral Phonon Scattering in FeVSb-Based Half-Heusler Compounds. EPL 2013, 104, 46003. 
(108) Xie, H.; Wang, H.; Pei, Y.; Fu, C.; Liu, X.; Snyder, G. J.; Zhao, X.; Zhu, T. Beneficial contribution of alloy disorder to electron and phonon transport in half-heusler thermoelectric materials. Adv. Funct. Mater. 2013, 23, 5123-5130.

(109) Kim, S. I.; Lee, K. H.; Mun, H. A.; Kim, H. S.; Hwang, S. W.; Roh, J. W.; Yang, D. J.; Shin, W. H.; Li, X. S.; Lee, Y. H.; Snyder, G. J.; Kim, S. W. Dense dislocation arrays embedded in grain boundaries for high-performance bulk thermoelectrics. Science 2015, 348, 109-114.

(110) Mingo, N.; Hauser, D.; Kobayashi, N. P.; Plissonnier, M.; Shakouri, A. "Nanoparticle-in-alloy" approach to efficient thermoelectrics: silicides in SiGe. Nano Lett. 2009, 9, 711-715.

(111) Lippmann, G.; Kästner, P.; Wanninger, W. Elastic constants of PbSe. Phys. Status Solidi A 1971, 6, K159-K161.

(112) Ravich, Y. I.; Efimova, B. A.; Smirnov, I. A., Semiconducting lead chalcogenides. Plenum Press: New York, 1970.

(113) Chattopadhyay, T.; von Schnering, H. G.; Grosshans, W. A.; Holzapfel, W. B. High pressure X-ray diffraction study on the structural phase transitions in $\mathrm{PbS}, \mathrm{PbSe}$ and $\mathrm{PbTe}$ with synchrotron radiation. Phys. BC 1986, 139, 356-360. 